\title{
$\beta$-Adrenergic Receptor-mediated Regulation of Extracellular Adenosine in Cerebral Cortex in Culture
}

\author{
Paul A. Rosenberg, Roger Knowles, Kelly P. Knowles, and Ya Li \\ Department of Neurology and Program in Neuroscience, Children's Hospital and Harvard Medical School, Boston, \\ Massachusetts 02115
}

Adenosine is an important inhibitory neuromodulator in the CNS, yet the sources of extracellular adenosine have yet to be well characterized. In this study we show that $\beta$-adrenergic stimulation of cortical cultures results in the extracellular accumulation of CAMP as well as adenosine, and that the extracellular adenosine derives from extracellular CAMP. The concentration dependence of isoproterenol in evoking CAMP secretion was determined by radioimmunoassay, and the $E C_{50}$ for this effect was found to be approximately $100 \mathrm{~nm}$. In order to investigate the effect of $\beta$-adrenergic stimulation on the regulation of extracellular adenosine, the effect of isoproterenol in stimulating the extracellular accumulation of adenine-containing compounds was examined by HPLC. Isoproterenol stimulated CAMP secretion in both astrocyte cultures and astrocyte-rich mixed cultures of astrocytes and neurons. However, no extracellular CAMP was detectable In neuron-enriched astrocytepoor cultures. Extracellular adenosine increased in response to isoproterenol in the astrocyte-rich mixed cultures, but not in the neuron-enriched astrocyte-poor cultures. After $\mathbf{3 0}$ min exposure to isoproterenol, the concentration of adenosine in the extracellular medium increased by $47 \%$ in 56 experiments in the mixed astrocyte-rich cultures.

In order to establish whether the adenosine that accumulates in response to isoproterenol stimulation actually derives from extracellular CAMP, phosphodiesterase inhibitors were tested for their ability to block isoproterenol-stimulated adenosine accumulation. Isobutylmethylxanthine (IBMX; 100

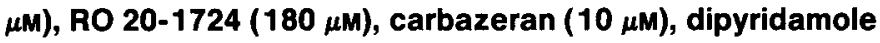
$(10 \mu \mathrm{M})$, and trifluoperazine $(10 \mu \mathrm{M})$ had no inhibitory effect on the isoproterenol-stimulated accumulation of extracellular adenosine. However $100 \mu \mathrm{M}$ IBMX plus $180 \mu \mathrm{M}$ RO 201724 effectively blocked isoproterenol-stimulated adenosine accumulation and, as expected, increased extracellular cAMP.

As a further test of the origin of isoproterenol-stimulated adenosine accumulation, we attempted to block this phenomenon by blocking CAMP secretion itself. For this purpose

Received Aug. 2, 1993; revised Oct. 15, 1993; accepted Oct. 26, 1993.

We thank Can Zhong and Sara Vasquez for excellent technical assistance, and Dr. Mohamad Mikati for helpful discussions early in this work. P.A.R. is supported by U.S. Public Health Service Grants NS26830 and NS31353, an Established Investigatorship from the American Heart Association, and a Mental Retardation Core Grant to Children's Hospital.

Correspondence should be addressed to Dr. Paul A. Rosenberg, Enders Research Building, Department of Neurology, Children's Hospital, 300 Longwood Avenue, Boston, MA 02115.

Copyright () 1994 Society for Neuroscience $0270-6474 / 94 / 142953-13 \$ 05.00 / 0$ probenecid, a known inhibitor of CAMP secretion in many different cell types, was used. We found that probenecid at $1 \mathrm{~mm}$ blocked isoproterenol-stimulated adenosine accumulation.

These studies suggest that one potentially important source of extracellular adenosine in the cerebral cortex is endogenous extracellular cAMP, secreted from astrocytes in response to $\beta$-adrenergic receptor stimulation. Since the receptors of neuromodulators other than norepinephrine may also be coupled to adenylyl cyclase in the cerebral cortex, there may be several neuromodulatory systems that regulate extracellular adenosine levels by this mechanism.

IKey words: norepinephrine, cAMP, phosphodiesterase, astrocytes, secretion, transport, $\beta$-adrenergic, isoproterenol]

Adenosine is an inhibitory modulator of central excitatory neurotransmission. Adenosine receptors in the CNS have been well characterized in ligand binding studies and have been mapped by autoradiography (Bruns et al., 1980; Goodman et al., 1983). Biochemical experiments have shown that adenosine blocks release of glutamate and aspartate from a number of preparations (Dolphin and Archer, 1983; Corradetti et al., 1984; Fastbom and Fredholm, 1985; Burke and Nadler, 1988), while electrophysiological experiments have shown that adenosine blocks excitatory postsynaptic events (Schubert and Mitzdorf, 1979; Dunwiddie, 1984; Okada and Ozawa, 1991; Scholz and Miller, 1991; Yoon and Rothman, 1991). Adenosine appears to block transmitter release by a presynaptic action limiting calcium influx (Wu et al., 1982; Proctor and Dunwiddie, 1983; Madison et al., 1987; Fredholm and Dunwiddie, 1988), and considerable evidence has been gathered showing that adenosine blocks calcium currents in peripheral (Dolphin et al., 1986; MacDonald et al., 1986) and central neurons (Proctor and Dunwiddie, 1983). Recently a strong case has been put forward that the effect of adenosine on excitatory transmitter release is mediated by an effect on calcium currents observable postsynaptically and mediated by the adenosine $A_{1}$ receptor (Scholz and Miller, 1991). Interestingly, both biochemical and physiological studies have shown that the effect of adenosine on synaptic neurotransmission is restricted to excitatory synapses (Dolphin and Archer, 1983; Yoon and Rothman, 1991). In addition, adenosine has been shown to activate a potassium conductance mechanism in hippocampal and striatal neurons (Trussel and Jackson, 1985; Scholz and Miller, 1991; Yoon and Rothman, 1991).

The sources of extracellular adenosine under physiological conditions in the CNS have not been established. Extracellular adenosine accumulation as a consequence of depolarization of 
neurons has been demonstrated many times in a variety of preparations (for review, see Dunwiddie, 1985). However, there still is considerable uncertainty about which cell types this extracellular adenosine derives from in each stimulation paradigm, whether adenosine itself is actually the species transported, and what the mechanisms of transport are. A substantial part of the difficulty arises from the fact that adenosine is a ubiquitous substance in cells. Thereforc, it is likcly that, for every cell type, conditions exist that result in the efflux of adenosine, for which a transport system is present in many cell membranes (Plagemann and Wohlhueter, 1980). The problem is determining how adenosine is transported under specific physiologic and pathologic conditions, and from what cell types under these conditions. For this purpose simple systems need to be studied using stimulus paradigms with simple rather than complicated effects on the tissue being studied. For an example of the problem, veratridine is often used as a depolarizing agent in studies of adenosine release, and unlike potassium depolarization, veratridine depolarization results in adenosine release that is not calcium dependent (MacDonald and White, 1985). However, veratridine causes not only depolarization but rapid depletion of cellular ATP stores through indirect activation of the $\mathrm{Na} / \mathrm{K}$ ATPase (Erecinska and Dagani, 1990), as well as neuronal swelling and death (Ramnath et al., 1992). Therefore, it is expected that there would be confusion concerning injurydependent release of adenosine, as opposed to physiological release, especially since both depolarization-associated toxicity and depolarization-evoked exocytotic release would be expected to be calcium dependent (Douglas and Rubin, 1963; Choi, 1987). It is doubtful whether veratridine stimulation experiments will tell us Inuch about the physiological release of adenosine; however, they might be a model for what happens in pathological states of oxygen or glucose deprivation in which tissue metabolic needs exceed the supply of energy, resulting in depletion of ATP and accumulation of intracellular adenosine.

In addition to these problems, many of the earlier studies looked at release of radiolabeled adenosine. Typically, tissues are preincubated with radioactive adenosine, which accumulates intracellularly as the nucleotides; the tissue is stimulated in some way, and efflux of radioactivity is measured. It is crucial to know what is the transported species (ATP or adenosine), and yet many studies have not addressed this issue. Furthermore, quantitation is impossible with radiolabel techniques because the specific activities of the released purines are unknown. Finally, there is always uncertainty whether radiolabeling techniques adequately label the pools that are most important for physiological activity. These problems have been addressed with the adoption of HPLC techniques for the measurement of adenine-containing compounds down to a subpicomolar level.

At this point it appears that we can identify several different possible mechanisms underlying adenosine release: (1) a calcium-dependent mechanism demonstrable in potassium-stimulated cortical slices (Hoehn and White, 1990a), synaptosomes (MacDonald and White, 1985), cerebellar neuronal cultures (Philibert and Dutton, 1989), and embryonic chick retinal neuronal cultures (Paes de Carvalho et al., 1990); (2) a calciumindependent mechanism that is demonstrable using veratridine stimulation (MacDonald and White, 1985), metabolic poisoning (Meghji et al., 1989), as well as glutamate receptor stimulation (both NMDA and non-NMDA receptors) (Hoehn and White, 1990c) and that probably utilizes the bidirectional nucleoside transporter (Meghji et al., 1989); (3) a glutamate uptake-activated mechanism demonstrable in synaptosomes (Hoehn and White, 1990b); and (4) a mechanism dependent upon the hydrolysis of extracellular ATP that is itself released as a consequence of calcium-dependent (potassium stimulation) and calcium-independent (veratridine stimulation) processes (White, 1978).

It is striking that for all the attention that adenosine release has received, so little has been made of the possibility that norepinephrine-stimulated cAMP secretion and extracellular hydrolysis to adenosine might be important in determining the extracellular concentration of adenosine. There is a great deal of evidence showing that astrocytes are an important target for the actions of norepinephrine (Stone and Ariano, 1989). It has been shown previously that norepinephrine stimulates intracellular cAMP accumulation in cortical cultures, and that this accumulation of cAMP occurs primarily in astrocytes. In addition, it was demonstrated that stimulation with isoproterenol caused the efflux of relatively large quantities of cAMP into the culture medium (Rosenberg and Dichter, 1989). This raised the question whether the secretion of cAMP might have a role in modulating the activity of nearby neurons by a paracrine mechanism. Since exogenous cAMP added to the medium was hydrolyzed to adenosine, one possible mechanism by which secretion of cAMP might influence nearby neurons would be if this cAMP were a significant source of adenosine (Rosenberg and Dichter, 1989). However, the demonstration of cAMP secretion and of metabolism of exogenous cAMP to adenosine does not establish that endogenously derived extracellular cAMP is a source of adenosine, and so the present investigations were undertaken to determine whether $\beta$-adrenergic stimulation of cultures could be shown to cause an increase in extracellular adenosine, and if so, whether such extracellular adenosine accumulation could be shown to be derived from extracellular cAMP.

\section{Materials and Methods}

Tissue culture. Astrocyte-rich, astrocyte-poor, and astrocyte cultures were prepared according to methods previously published (Rosenberg and Aizenman, 1989; Rosenberg, 1991). Astrocyte-rich and astrocytepoor mixed (neurons plus glia) cultures were derived from embryonic day $16 \mathrm{CD}$ rat embryos (Charles River). Astrocyte-rich cultures were subjected to mitotic inhibition using cytosine arabinoside when glial cells became confluent, at $15 \mathrm{~d}$ in vitro (DIV). Astrocyte-poor cultures were subjected to mitotic inhibition at $4 \mathrm{DIV}$. Astrocyte-rich cultures contained $90-95 \%$ astrocytes, whereas astrocyte-poor cultures contained approximately $30 \%$ non-neuronal cells, mostly astrocytes. Astrocytepoor cultures were used from 18 to 40 DIV. Astrocyte-rich cultures were used from 24 to 56 DIV. Astrocyte cultures were used from 24 to 56 DIV. Astrocyte cultures were prepared from postnatal day 1 animals according to methods previously published (Rosenberg and Dichter, 1989).

Isoproterenol stimulation. Cultures were washed in physiological saline and then placed in Earle's salt solution without phenol red (ESS), $1.2 \mathrm{ml} / 35 \mathrm{~mm}$ dish, with slow rotary agitation at $36^{\circ} \mathrm{C}$ in $95 \%$ air, $5 \%$ $\mathrm{CO}_{2}$ for $4 \mathrm{hr}$. We found that medium change itself produces a significant release of adenosine into the medium, which then declines and reaches a plateau by approximately $2 \mathrm{hr}$; therefore, we used a 2-4 hr preincubation period for our stimulation experiments. At zero time, isoproterenol was added from a $100 \times$ stock in $1 \mathrm{mM} \mathrm{HCl}$. Controls received $1 \mathrm{mM} \mathrm{HCl}$ only. Buffering was adequate in ESS to prevent a significant $\mathrm{pH}$ change (less than 0.1 unit). Catecholamines are relatively stable in $1 \mathrm{~mm} \mathrm{HCl}$, and stock solutions can be kept frozen for weeks without significant oxidation. At selected times, $1 \mathrm{ml}$ of medium was removed from the culture dish, made $10 \mathrm{~mm}$ in EDTA, and then placed in a boiling water bath for $3 \mathrm{~min}$.

Derivatization of samples to form the etheno derivatives. We used the method of Perrett (1987) for the determination of adenine-containing 
Table 1. The elution times of selected adenine-containing compounds

\begin{tabular}{lrrr} 
& & & \multicolumn{1}{l}{$\begin{array}{l}k^{\prime} \\
\text { (Per- } \\
\text { rett, } \\
\text { Compound }\end{array}$} \\
\hline 2',5'-Dideoxyadenosine & $4.49 \pm 0.18$ & $2.73 \pm 0.06$ & \\
Adenine & $4.57 \pm 0.17$ & $2.55 \pm 0.23$ & 2.99 \\
NADP & $4.7 \pm 0.04$ & $2.9 \pm 0.06$ & 3.40 \\
AMP & $4.84 \pm 0.04$ & $3.07 \pm 0.09$ & 3.14 \\
NAD & $5.45 \pm 0.06$ & $3.51 \pm 0.09$ & \\
ADP & $5.81 \pm 0.03$ & $3.97 \pm 0.12$ & 4.09 \\
Adenosine & $6.85 \pm 0.06$ & $4.76 \pm 0.13$ & 4.84 \\
DeoxyAMP & $8.33 \pm 0.31$ & $5.92 \pm 0.26$ & 5.08 \\
Deoxyadenosine & $8.73 \pm 0.19$ & $6.25 \pm 0.16$ & 6.43 \\
ATP & $8.92 \pm 0.12$ & $6.65 \pm 0.07$ & 7.09 \\
2,3-cAMP & $9.21 \pm 0.08$ & $6.6 \pm 0.14$ & \\
Cyclic deoxyadenosine & & & \\
$\quad$ monophosphate & $16.54 \pm 0.12$ & $12.9 \pm 0.3$ & \\
Cyclic adenosine & & & \\
$\quad$ monophosphate & $22.58 \pm 0.79$ & $17.98 \pm 0.9$ & 18.56
\end{tabular}

The values represent the mean and SD from at least three separate determinations. $k^{\prime}$ is the capacity factor $\left[k^{\prime}=\left(t_{r}-t_{m}\right) / t_{m}\right.$, where $t_{m}$ is the retention time of the mobile phase and $t_{r}$ is the retention time of the compound of interest].

compounds by rcversed-phase ion-pair high-performance liquid chromatography. This method achieves the separation of the 1-N6-etheno derivatives of many adenine-containing compounds. Derivatization to form the etheno derivatives was accomplished by a simple precolumn step in which the samples were heated with chloracetaldehyde (Secrist et al., 1972). Samples were derivatized immediately or after $24 \mathrm{hr}$ storage at $4^{\circ} \mathrm{C}$. In initial experiments, $100 \mu$ l of $2 \mathrm{M}$ potassium phosphate (pH 6.0 ), and $50 \mu \mathrm{l}$ of $55 \%$ chloracetaldehyde (Fluka) were added, mixed, and placed in a boiling water bath for a 10 min period, after which the reaction was stopped by removing the tubes to an ice bath. Samples were then spun at $14,000 \mathrm{rpm}$ in an Eppendorf microcentrifuge. Derivatized samples were stored at $4^{\circ} \mathrm{C}$ until assay, which was within $72 \mathrm{hr}$. In later experiments, the $\mathrm{pH}$ of the sample was lowered using potassium phosphate at pH 5.6 and adding $50 \mu \mathrm{l}$ of $1 \mathrm{M} \mathrm{HCl}$ to the sample. Final $\mathrm{pH}$ was 5.6. Fifty microliters of a $10 \%$ solution of chloracetaldehyde were used, and samples were boiled for $45 \mathrm{~min}$. These modifications of the procedure considerably decreased the presence of background fluorescence in the samples and increased the yield of formation of the etheno derivatives.

Table 1 shows the retention times and capacity factors obtained in at least three chromatographic analyses for various adenine-containing compounds using this procedure. Based on this data, we determined that cyclic deoxyadenosine monophosphate would be a useful compound as an internal standard in the HPLC assay, given the chemical similarity between it and the compounds of interest in these studies, and its elution time, which is significantly different from the other compounds of interest. The use of an internal standard controls for several possible sources of measurement error: pipetting error, variation in derivatization yicld, variation in lamp intensity, variation in photomultiplier sensitivity, and variation in recorder sensitivity. Cyclic deoxyadenosine monophosphate was therefore used as an internal standard in some experiments, added to each sample to achieve a final concentration of $8.3 \mathrm{pmol} / 50 \mu \mathrm{l}$.

Yield. In six experiments the following yields of the etheno compounds were obtained starting with the indicated adenine-containing compounds: adenosine, $77.1 \pm 2.5 \%$; AMP, $82.4 \pm 4.9 \%$; cAMP, 89.3 $\pm 9.3 \%$.

Chromatography. Standards of adenosine, AMP, and cAMP were derivatized and run with samples in each experiment. Complete standard curves for each substance were run with the samples from each experiment, with standards bracketing the range in which the compounds appeared in the samples. Absolute quantities of adenine-containing compounds were determined by comparing peak heights of samples with those of standards. In samples run with the internal standard, the ratios of peak heights in the samples to the peak height of the internal standard were compared with the same for standards. 1-N6-etheno standards (Sigma) for each compound were also run in each set of samples in order to calculate yield for the derivatization procedure for each compound in cach experiment.

Samples were analyzed using a $50 \mu \mathrm{l}$ loop. An isocratic elution system was used with $10 \%$ methanol, $50 \mathrm{~mm}$ ammonium acetate, $1 \mathrm{~mm}$ EDTA, $0.2 \mathrm{~mm}$ tetrabutylammonium hydrogen sulfate, $\mathrm{pH} 5.6$ as the running buffer; flow rate $=1 \mathrm{ml} / \mathrm{min}$. A C18 silica column was used ( $3 \mu \mathrm{m}$ ODS Hypersil, Keystone Scientific, Inc., Bellefonte, PA). Fluorescence of the eluate was monitored using a fluorescence detector (Schoeffel model FS 970) equipped with a xenon lamp, a monochromator (Schoeffel GM 970 ), and a $>360$ emission filter. Excitation wavelength was $212 \mathrm{~nm}$. In some experiments, a McPherson fluorescence detector with a $200 \mathrm{~W}$ xenon-mercury lamp was used with excitation at the mercury emission band at $265 \mathrm{~nm}$. Data was acquired on chart recorders, and peak heights were measured manually in initial experiments. Subsequently, data were acquired and analyzed using System Gold software from Beckman Instruments and a Beckman model 406 analog-to-digital interface.

Verification of identity of peaks. Verification of the identification of peaks was accomplished by three criteria: (1) identity of elution times, (2) coelution of added compound with the putatively identified compound, and (3) elimination of the peak with incubation of sample with adenosine deaminase (for adenosine) and cyclic nucleotide phosphodiesterase (for cAMP).

For identification of the cAMP peak, $3^{\prime}: 5^{\prime}$-cyclic nucleotide phosphodiesterase from beef heart was used (Sigma P-0134); $0.012 \mathrm{U}$ was added to a $300 \mu$ sample (without EDTA) and incubated for 3 hr at $37^{\circ} \mathrm{C}$. This procedure specifically eliminated the putative cAMP peak.

For identification of the adenosine peak, adenosine deaminase from calf intestine was used (Boehringer-Mannheim 102 091); $2.0 \mathrm{U}$ was added to a $500 \mu \mathrm{l}$ sample, which was then incubated for $3 \mathrm{hr}$ at $37^{\circ} \mathrm{C}$. This proccdure specifically eliminated the putative adenosine peak.

The identification of AMP was more problematic. There was a large peak that eluted with a retention time very close if not identical to that of AMP. When authentic AMP was added to the sample, in some runs it coeluted with this peak, suggesting that it might be AMP. However, we found that tryptophan, which is a fluorescent amino acid that is present in the growth medium, and which is apparently secreted by cells in the cultures, also coeluted with this peak. Because of the difficulty in resolving AMP from tryptophan, in this study we have not attempted to quantitate AMP.

Radioimmunoassay of CAMP. Cyclic AMP was assayed by radioimmunoassay kit from New England Nuclear, using methods previously published (Rosenberg and Dichter, 1989). These experiments were conducted in 24-well plates, using one glass coverslip per well (transferred to the wells at the start of the experiment), and $0.5 \mathrm{ml}$ of ESS per well. In these experiments (data shown in Figs. 1, 2), the quantity of tissue to medium volume $(100 \mathrm{mg} / 500 \mu \mathrm{l})$ was considerably less than in the later experiments (Figs. 3-10), which were assayed by HPLC, and in which the tissue to medium volume was $850 \mathrm{mg} / 1200 \mu \mathrm{I}$.

At the appropriate times, $400 \mu \mathrm{l}$ samples were removed from wells. Zero time point samples were treated the same as other samples but were not exposed to cells. Samples were placed in $400 \mu \mathrm{l}$ of ice-cold 0.6 $\mathbf{M ~ H C l O}$ and placed on ice for $15 \mathrm{~min}$. Eighty microliters of $3 \mathbf{M ~} \mathrm{KHCO}_{3}$ were added for $30 \mathrm{~min}$. This procedure resulted in a dilution of the medium cAMP to 0.45 times the original concentration. Tubes were centrifuged for $1 \mathrm{~min}$ and $700 \mu \mathrm{l}$ was decanted and stored at $-20^{\circ} \mathrm{C}$ until cAMP concentration of each sample was assayed.

The difference in tissue mass/medium volume in the experiments assaycd by radioimmunoassay and by HPLC, together with the dilution of the sample during preparation in the experiments assayed by radioimmunoassay, would account for an eightfold difference between the concentrations of cAMP actually measured in the two sets of experiments. In fact, differences of this order of magnitude in medium cAMP concentration in the two sets of experiments were observed, as seen in the medium cAMP concentrations presented in Figures 1 and 2 compared with those presented in Figures 3-10.

Chemicals. Adenine-containing compounds and their etheno adducts as well as all other compounds were obtained from Sigma. HPLC-grade methanol and chloracetaldehyde ( $55 \%$ solution) were obtained from Fluka. Rolipram was obtained from Schering AG, Berlin, West Germany. RO 20-1724 was obtained from Calbiochem. Carbazeran citrate was obtained from Pfizer Central Research, Kent, UK.

Statistics. Tests of significance werc performed using the INSTAT program from GraphPad Sofware. Data were subjected to an analysis of variance (ANOVA) followed by a Tukey-Kramer multiple comparisons 

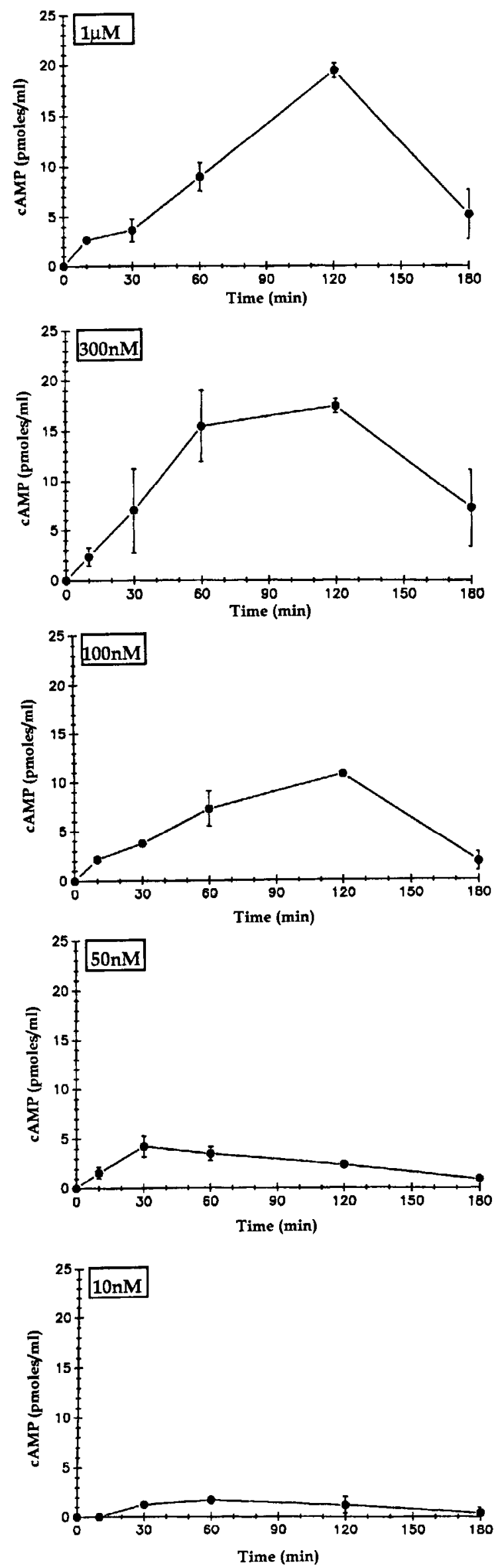

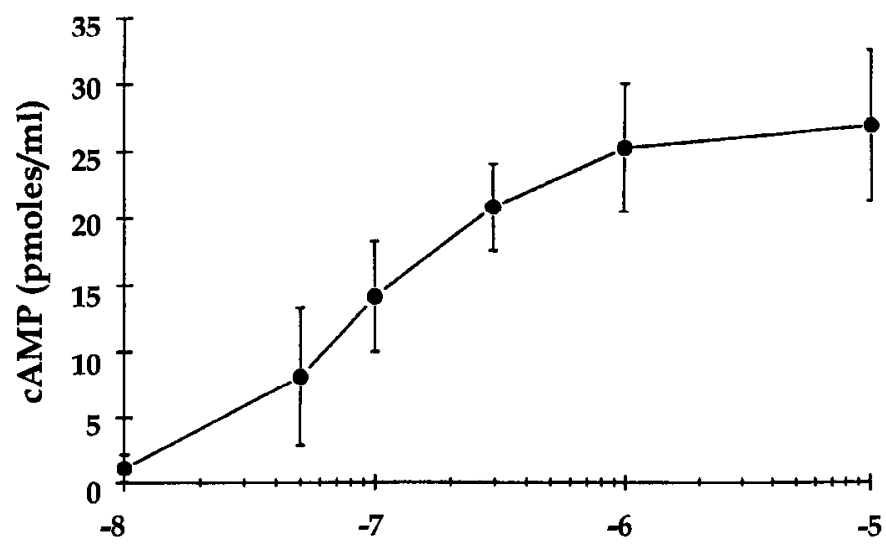

\section{LOG [ISOPROTERENOL]}

Figure 2. Duse-response relationship for isoproterenol-stimulated secretion of cAMP. The results from five experiments of the type displayed in Figure 1 were pooled, and the peak concentrations of cAMP attained at each concentration of isoproterenol were plotted. Means plus SD are shown. A given point represents two to five individual values because the selected concentrations were not the same in all experiments. The $\mathrm{EC}_{50}$ for this pooled data was $100 \mathrm{nM}$.

test. The parametric assumption was tested by Bartlett's test for homogeneity of variances. When a nonparametric test of significance was required, the Kruskal-Wallis nonparametric ANOVA was used, followed by Dunn's multiple comparisons test.

\section{Results}

Concentration dependence of effect of isoproterenol on $C A M P$ accumulation

Previously, we showed that $10 \mu \mathrm{M}$ isoproterenol produced intracellular and extracellular cAMP accumulation in cortical cultures (Rosenberg and Dichter, 1989). Figure 1 shows a doseresponse experiment in which at each of several concentrations of isoproterenol the secretion of cAMP from astrocyte-rich cultures was measured by radioimmunoassay at selected time points during continuous exposure to the drug. The radioimmunoassay results were expressed as concentrations, and the units given in Figures 1 and 2 for the $y$-axis are picomoles cAMP per milliliter of culture medium treated with perchloric acid and potassium bicarbonate as described in Materials and Methods (resulting

Figure 1. Time course of cAMP secretion at varying concentrations of isoproterenol. Astrocyte-rich cortical cultures were exposed to varying concentrations of isoproterenol from $10 \mathrm{nM}$ to $1 \mu \mathrm{M}$ and the appearance of cAMP in the extracellular medium was measured by radioimmunoassay. An effect of isoproterenol was detectable at $10 \mathrm{~nm}$ isoproterenol. Maximal effect was attained at $1 \mu \mathrm{M}$. The time to the peak response (maximal extracellular concentration of cAMP) was different at different concentrations. Coverslip cultures of rat cerebral cortex at 34 DIV were exposed to selected concentrations of isoproterenol, prepared by serial dilutions into ESS. Isoproterenol was prepared in a $10 \mathrm{~mm}$ stock in 1 mM $\mathrm{HCl}$ and stored at $-20^{\circ} \mathrm{C}$. Coverslip cultures were placed in ESS in a 24-well plate for $20 \mathrm{~min}$ to preincubate, and then medium was replaced with $0.5 \mathrm{ml}$ of medium plus isoproterenol. All the coverslips from one dish were exposed to one concentration of isoproterenol. Plates were placed on a rotary shaker at $36^{\circ} \mathrm{C}$ in a $5 \% \mathrm{CO}_{2}$ incubator. The resulting data were plotted (means $\pm \mathrm{SD}$ ) without further manipulation and are shown in Figures 1 and 2. Samples were run in duplicate. Each point shown represents data from a single coverslip. The figure represents data obtained from a single experiment using coverslips from five dishes. 
in a 0.45 dilution of the actual concentration of (AMP). cAMP secretion appears to be maximal at $1 \mu \mathrm{M}$, but an effect is detectable at concentrations as low as $10 \mathrm{~nm}$ isoproterenol. The peak concentrations of cAMP attained in a set of five time course experiments using concentrations of isoproterenol from $10 \mathrm{nM}$ to $10 \mu \mathrm{M}$ were chosen and plotted [the time course of the effect of $10 \mu \mathrm{M}$ isoproterenol on extracellular cAMP accumulation has been shown previously (Rosenberg and Dichter, 1989); in the particular experiment shown in Fig. 1, a $10 \mu \mathrm{M}$ category was not included], and the results of these experiments were pooled, generating the dose-response curve shown in Figure 2. The $\mathrm{EC}_{50}$ for the effect of isoproterenol on cAMP secretion in these experiments was approximately $100 \mathrm{nM}$. In all subsequent experiments, $1 \mu \mathrm{M}$ isoproterenol was used to stimulate cortical cultures.

\section{Assay of adenine nucleotides}

The specific experimental question that we addressed was whether $\beta$-adrenergic receptor stimulation of cortical cultures would result in a detectable increase in extracellular adenosine. For this experiment it was necessary to assay not only cAMP but also adenosine. To accomplish this, we used chloracetaldehyde derivatization of adenine compounds to form their fluorescent etheno derivatives (Secrist et al., 1972; Perrett, 1987), with separation by ion-pair reverse-phase HPLC on a C 18 column, using fluorescence detection (Perrett, 1987). Using this method, we determined the effect of stimulation of astrocyte-rich cortical cultures with $1 \mu \mathrm{M}$ isoproterenol on the appearance of cAMP and adenosine in the medium. Figure $3 \mathrm{~A}$ shows a chromatogram of a sample containing known concentrations of adenosine, AMP, and $\operatorname{cAMP}(2.5,2.5$, and $5 \mathrm{pmol} / 50 \mu \mathrm{l}$, rcspectively), which were derivatized and assayed by HPLC together with the test samples shown in Figure 3, $B$ and $C$. Figure $3 A$ is shown in order to indicate the elution times of the peaks of adenosine, AMP, and cAMP. Figure $3 B$ shows the chromatogram of a media sample taken from a control culture that was not exposed to drug. In this sample, adenosine but not cAMP was present. Figure $3 C$ shows a chromatogram of a medium sample taken from cultures that were exposed to $1 \mu \mathrm{M}$ isoproterenol for $30 \mathrm{~min}$. Following exposure to $1 \mu \mathrm{M}$ isoproterenol, cAMP appeared in the medium, and adenosine was increased, in this experiment to $214 \%$ of the control value.

In Figure 3, $B$ and $C$, the adenosine and cAMP peaks have been tentatively identified by the similarity of their elution time to the elution time of the authentic standards shown in Figure $3 A$. Two additional criteria were used in order to make these identifications with certainty: coclution of authentic compound with the putatively identified compound in the sample and elimination of the peak by enzymatic digestion (see Materials and Methods)

\section{Reproducibility of phenomenon}

The experiments described in this report extended over a period of 29 months. In this time, a total of 56 experiments were performed in cultures $24-56$ DIV in which the adenosine concentration was measured in the extracellular medium of cortical cultures exposed to $1 \mu \mathrm{M}$ isoproterenol for $30 \mathrm{~min}$ and compared to control cultures. In 49 of these experiments, the mean of the isoproterenol-treated group was greater than the mean of the control group. The mean adenosine concentration in the control cultures was $1.5 \pm 1 \mathrm{pmol} / 50 \mu \mathrm{l}(30 \pm 20 \mathrm{nM} ; n=155$ determinations). The mean adenosine concentration in the isopro-
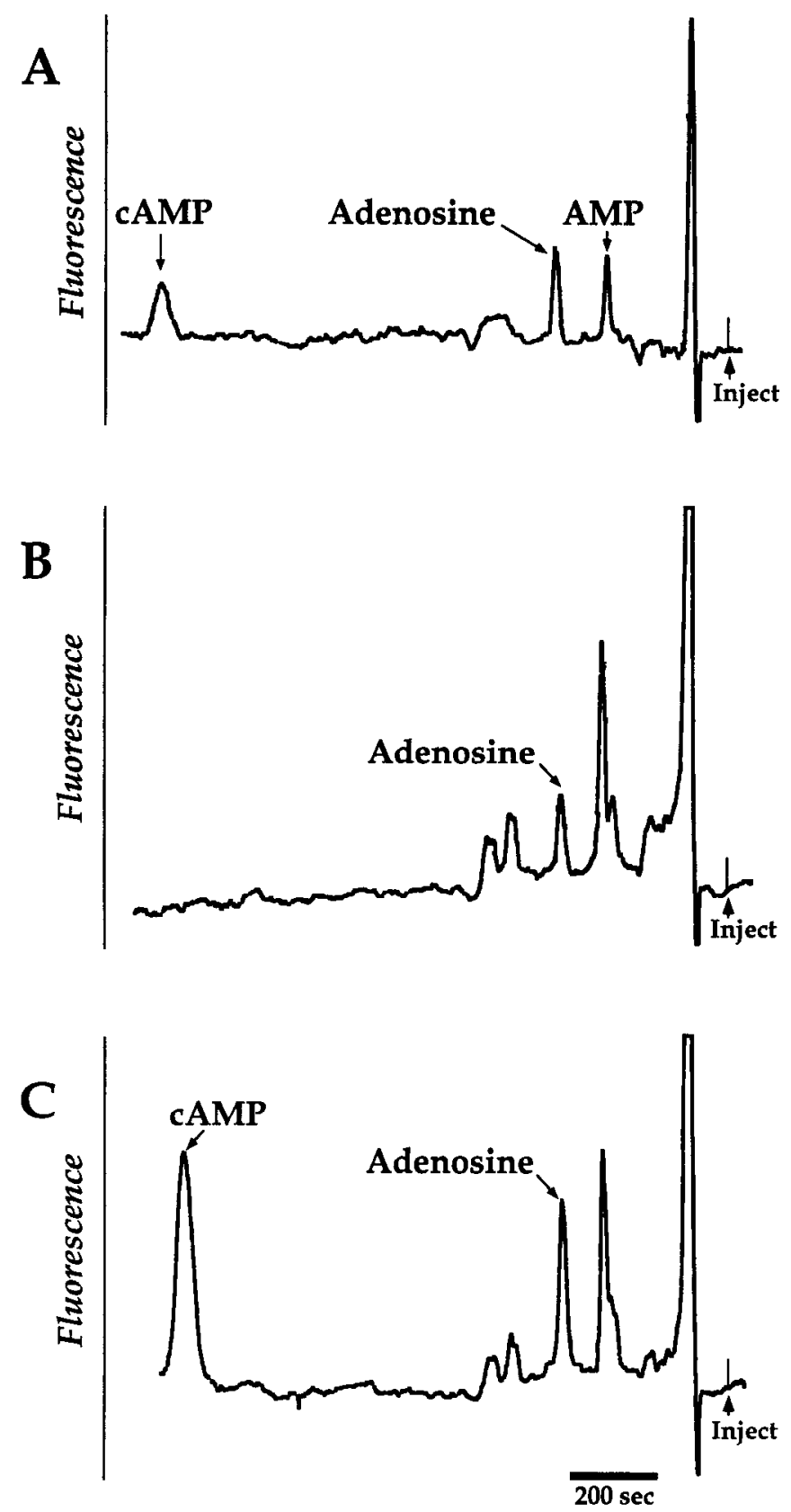

Figure 3. Elution profile of adenine-containing compounds present in medium of cortical cultures exposed to isoproterenol: sample chromatograms of a standard solution containing $2.5 \mathrm{pmol}$ adenosine, 2.5 pmol AMP, and 5 pmol cAMP per $50 \mu \mathrm{l}$, derivatized prior to assay $(A)$; a sample of control medium from astrocyte-rich cultures incubated for $30 \mathrm{~min}$ with vehicle only, derivatized prior to assay $(B)$; and a sample of medium from astrocyte-rich cultures incubated with $1 \mu \mathrm{M}$ isoproterenol for $30 \mathrm{~min}$, derivatized prior to assay $(C)$. Adenine-containing compounds in the samples were assayed by HPLC with fluorescence detection. Isoproterenol causes the appearance of a large peak that migrates with the same retention time as CAMP, and causes an increase in the peak that has the same elution time as adenosine. In addition, there is a peak that migrates with the retention time of AMP, and that does not seem to be affected by the presence of isoproterenol. Fluorescence of the eluate was monitored using a Schoeffel fluorometer with monochromator at $212 \mathrm{~nm}$. Fluorescence intensity is given in arbitrary units. Arrows display time of injection of samples. Data were digitized directly from the chart paper; recorder was run at $0.2 \mathrm{~mm} / \mathrm{sec}$ with sensitivity of $5 \mu \mathrm{V} / \mathrm{mm}$. 

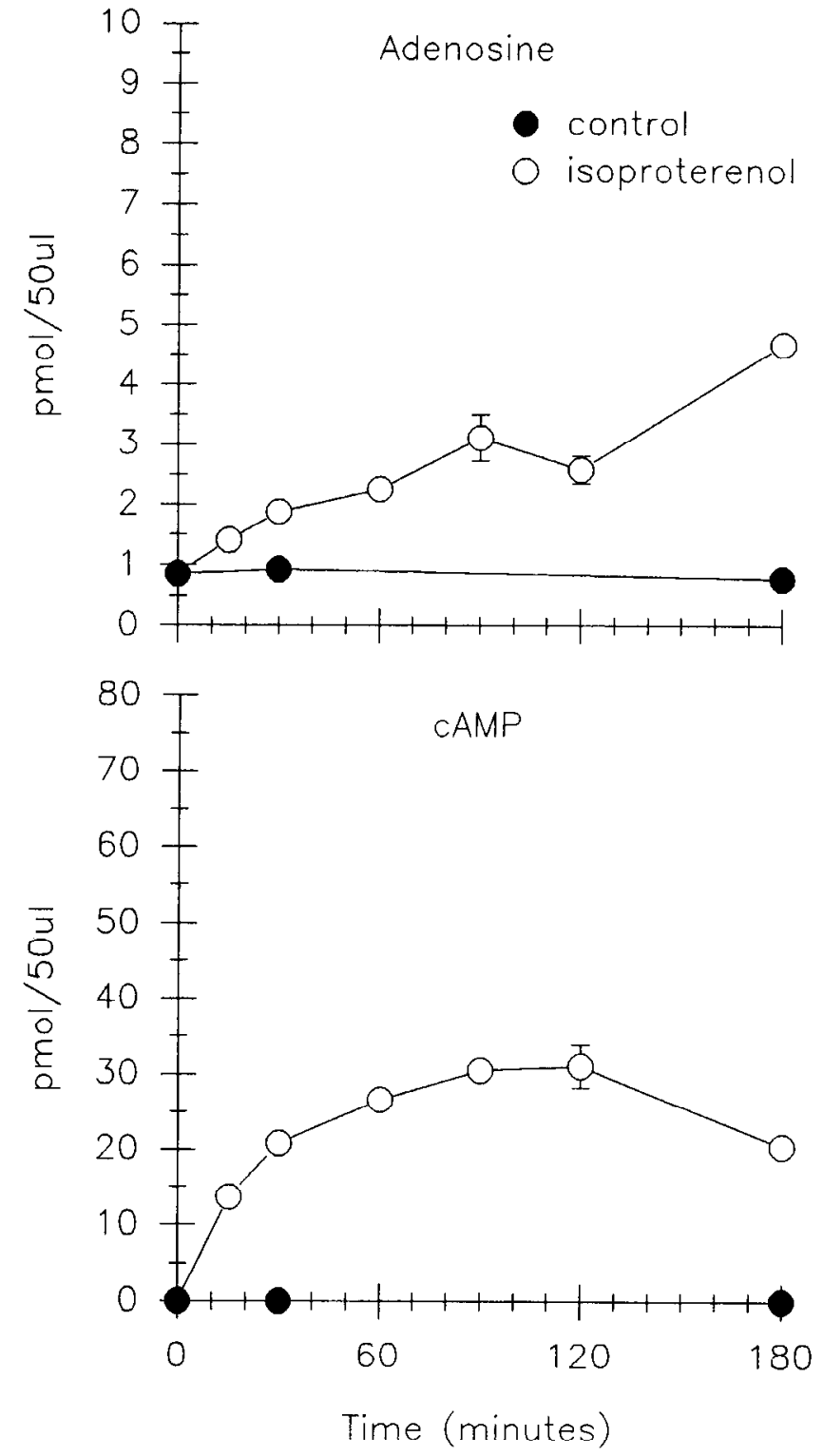

Figure 4. Time course of appearance of adenosine and cAMP in the extracellular medium of astrocyte-rich mixed cultures of astrocytes and neurons following stimulation by $1 \mu \mathrm{M}$ isoproterenol. Astrocyte-rich cultures (39 DIV) were exposed to $1 \mu \mathrm{M}$ isoproterenol and media samples were taken at selected time points and assayed by HPLC. cAMP attains a maximum between 60 and $120 \mathrm{~min}$ and then declines. At the maximum, cAMP in the extracellular medium was $640 \mathrm{nM}$. Adenosine demonstrated a timc-dcpendent and continuous incrcasc in the presence of isoproterenol. The baseline concentration was $17 \mathrm{nM}$, and this increased over $120 \mathrm{~min}$ to $52 \mathrm{~nm}$.

terenol-treated cultures was $2.2 \pm 1.3 \mathrm{pmol} / 50 \mu \mathrm{l}(44 \pm 26 \mathrm{nM}$; $n=159$ determinations; $p<0.0001$ ), a $47 \%$ increase.

We investigated the time course of isoproterenol-stimulated extracellular adenosine and cAMP accumulation in order to characterize these phenomena further, including the relationship of the adenosine accumulation to cAMP transport. Our previous work suggested that $\beta$-adrenergic receptors in cortical cultures are localized primarily on astrocytes (Rosenberg and Dichter, 1989). Furthermore, preliminary studies suggested to us that an extracellular cyclic nucleotide phosphodiesterase is present in cortical cultures (Rosenberg and Dichter, 1989; Vasquez and Rosenberg, 1989), and that it appeared to be localized primarily to neurons (Vasquez and Rosenberg, 1989). In order to understand the role of astrocytes and neurons in these phenomena, time course experiments were conducted on astrocyterich cultures, composed of 90-95\% neurons; astrocyte-poor cultures, which are $20-30 \%$ astrocytes [containing approximately $1 / 50$ the number of astrocytes as in the astrocyte-rich cultures (Rosenberg, 1991)]; and astrocyte cultures.

\section{Assay of adenine nucleotides in astrocyte-rich cultures stimulated with isoproterenol}

Figure 4 shows a time course experiment plotting cAMP and adenosine appearing in the medium of astrocyte-rich cultures (39 DIV, containing $740 \pm 90 \mathrm{mg}$ of protein) stimulated with $1 \mu \mathrm{M}$ isoproterenol. cAMP secretion achieved a peak concentration of $32 \mathrm{pmol} / 50 \mu \mathrm{l}$ or $640 \mathrm{~nm}$, at $60-120 \mathrm{~min}$, subsequently declining, as has been noted before (Rosenberg and Dichter, 1989). Adenosine continuously increased in response to isoproterenol stimulation, in this experiment from a basal concentration of 17-52 $\mathrm{nM}$ after $120 \mathrm{~min}$ exposure to isoproterenol. In seven time course experiments the extracellular adenosine concentration rose with isoproterenol from a basal level of $10 \pm 6$ $\mathrm{nM}$ to $41 \pm 19 \mathrm{nM}$ at $120 \mathrm{~min}(p<0.002)$.

\section{Assay of adenine nucleotides in astrocyte cultures stimulated with isoproterenol}

For comparison with the response to isoproterenol in astrocyterich cultures, Figure 5 shows a similar time course experiment but using astrocyte cultures (41 DIV), with a protein content of $440 \pm 60 \mathrm{mg} /$ dish. Here extracellular cAMP accumulation was also seen, and was in fact greater than that observed with the culturcs containing ncurons. In this experiment, basal extracellular adenosine was $5 \mathrm{nM}$ and rose to $9 \mathrm{nM}$ after $120 \mathrm{~min}$ exposure to isoproterenol. In six experiments with astrocyte cultures, extracellular adenosine after exposure to isoproterenol for $120 \mathrm{~min}(11 \pm 7 \mathrm{nM})$ was not significantly different from basal levels $(7 \pm 4 \mathrm{~nm})$.

Extracellular cAMP concentrations found in the media of astrocyte-rich and astrocyte cultures exposed to isoproterenol were compared. No cAMP was detected in the absence of isoproterenol stimulation. In astrocyte-rich cultures, following 60 min stimulation, the extracellular cAMP concentration was 482 $\pm 208 \mathrm{~nm}(n=7)$. In astrocyte cultures, following $60 \mathrm{~min}$ stimulation, the extracellular cAMP concentration was $904 \pm 468$ $\mathrm{nM}(n=6)$.

\section{Assay of adenine nucleotides in neuron-enriched, astrocyte- poor cultures stimulated with isoproterenol}

Figure 6 shows an experiment using neuron-enriched astrocytepoor cultures (19 DIV), with a protein content of $140 \pm 10 \mathrm{mg}$ / dish. Here we saw no cAMP accumulation, consistent with previous work (Rosenberg and Dichter, 1989) and with the fact that relatively few astrocytes are present. In addition, no significant change in adenosine was observed from the basal level, which in this experiment was $36 \mathrm{~nm}$. In five experiments, we found a basal concentration for adenosine in astrocyte-poor cultures of $55 \pm 22 \mathrm{nM}$. After exposure to isoproterenol for 120 $\min$, the adenosine concentration was $61 \pm 27 \mathrm{~nm}$.

The demonstration that isoproterenol stimulated the secretion of cAMP and also caused an increase in the extracellular concentration of adenosine suggested that the increase in adenosine had extracellular cAMP as its source. The relationship between cAMP secretion and extracellular adenosine is impor- 

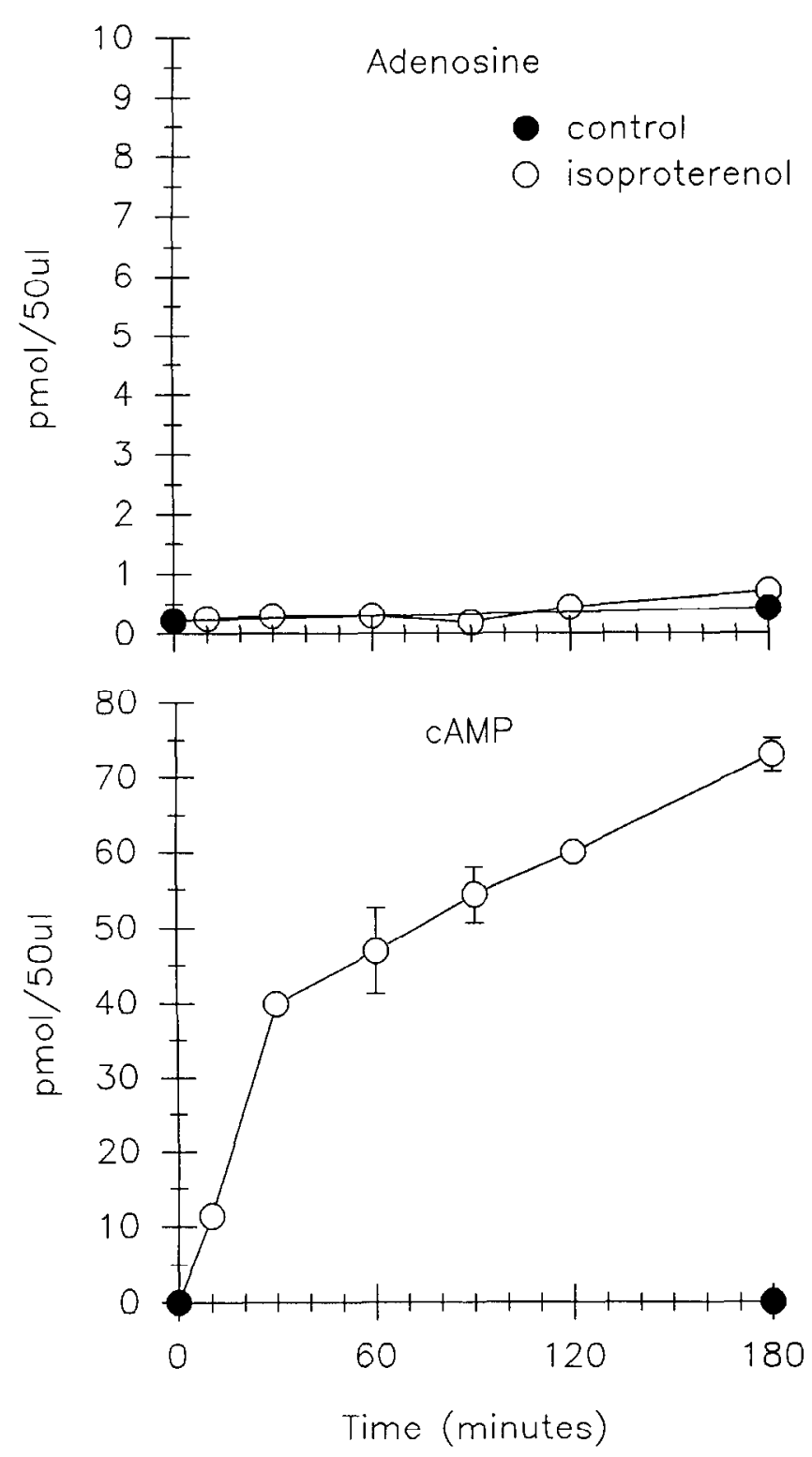

Figure 5. Time course of appearance of adenosine and cAMP in the extracellular medium of astrocyte cultures following stimulation by 1 $\mu \mathrm{M}$ isoproterenol. Astrocyte cultures (41 DIV) were exposed to $1 \mu \mathrm{M}$ isoproterenol, and medium was sampled at selected time points. No significant effect of isoproterenol on adenosine accumulation was observed. cAMP progressively accumulates over the time course of this experiment, and reaches $1.4 \mu \mathrm{M}$.

tant to our understanding of the regulation of extracellular adenosine concentration, and so we undertook to establish whether the increase in adenosine that appears to be a consequence of $\beta$-adrenergic stimulation in fact derives from secreted cAMP. Two approaches to establishing the relationship between extracellular cAMP and extracellular adenosine that we have taken rely on inhibition of cyclic nucleotide phosphodiesterase and inhibition of cAMP secretion.

\section{Effect of cyclic nucleotide phosphodiesterase inhibitors on isoproterenol-stimulated adenosine accumulation}

We tried to block the isoproterenol-stimulated increase in extracellular adenosine using phosphodiesterase inhibitors. If formation of adenosine were dependent upon hydrolysis of cAMP
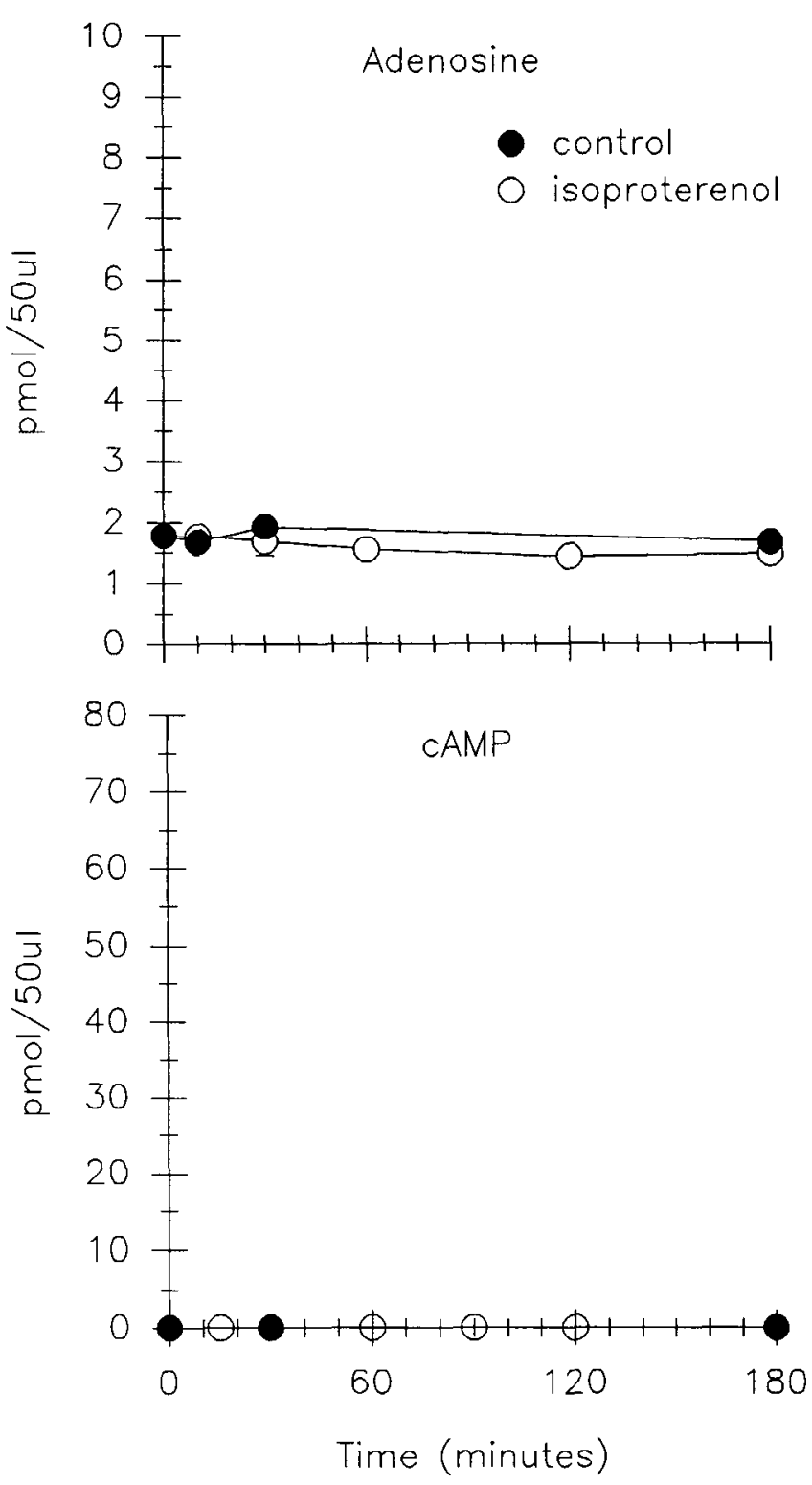

Figure 6. Time course of appearance of adenosine and cAMP in the extracellular medium of neuron-enriched astrocyte-poor cultures following stimulation by $1 \mu \mathrm{M}$ isoproterenol. Neuron-enriched astrocytepoor cultures (19 DIV) were exposed to $1 \mu \mathrm{M}$ isoproterenol, and medium was sampled at selected time points. No effect on adenosine or cAMP accumulation was observed.

by phosphodiesterase, then inhibition of phosphodiesterase should prevent an increase in adenosine. In order to pursue this test of the hypothesis that isoproterenol-stimulated extracellular adenosine accumulation derives from extracellular cAMP, we tested a variety of cyclic nucleotide phosphodiesterase inhibitors: isobutylmethylxanthine (IBMX), a nonspecific cyclic nucleotide phosphodiesterase inhibitor (Beavo, 1988); RO 20-1724, an inhibitor of calcium-independent cAMP-specific phosphodiesterase prevalent in the brain (Beavo, 1988; Beavo and Reifsnyder, 1990; Challiss and Nicholson, 1990; Nicholson et al., 1991); carbazeran, an inhibitor of cGMP-inhibited phosphodiesterase (Weishaar et al., 1986); dipyridamole, an inhibitor of cGMP-specific phosphodiesterase (Weishaar et al., 1986); and trifluoperazine, an inhibitor of calcium/calmodulin-dependent phosphodiesterase (Levin and Weiss, 1977). However, we found 


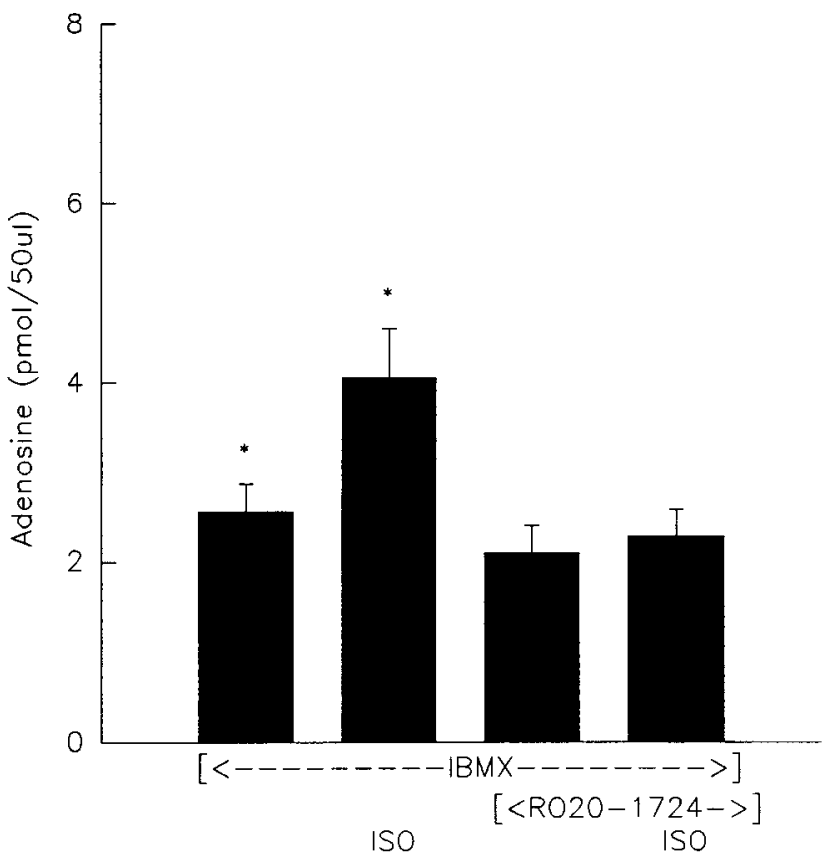

Figure 7. Effect of IBMX plus RO 20-1724 on isoproterenol-stimulated adenosine accumulation. RO 20-1724 plus IBMX block isoproterenol-stimulated adenosine accumulation. Cultures were incubated with or without $1 \mu \mathrm{M}$ isoproterenol for $30 \mathrm{~min}$ in the presence of IBMX $100 \mu \mathrm{M}$ and with or without $180 \mu \mathrm{M}$ RO 20-1724. The results of 15 experiments were pooled. Error bars show SEM. In the presence of IBMX, isoproterenol produced a significant $(p<0.05)$ increase in extracellular adenosine (asterisks). This effect was eliminated by the inclusion of RO 20-1724.

that several of these inhibitors by themselves, in the absence of isoproterenol, caused an increase in extracellular adenosine. Therefore, we restricted ourselves to working with concentrations of phosphodiesterase inhibitors that produced a doubling or less of the baseline concentrations of adenosine. We found that IBMX at $100 \mu \mathrm{M}(n=30$ experiments; see, e.g., Fig. 7), RO 20-1724 (180 $\mu \mathrm{M}, n=9)$, dipyridamole $(10 \mu \mathrm{M}, n=2)$, carbazeran citrate $(10 \mu \mathrm{M}, n=3)$, and trifluoperazine $(10 \mu \mathrm{M}, n$ $=2$ ) failed to block the effect of isoproterenol on extracellular adenosine.

In 15 more experiments we combined the use of $100 \mu \mathrm{M}$ IBMX with $180 \mu \mathrm{M}$ RO 20-1724. We found that when cortical cultures were exposed to RO 20-1724 together with IBMX, isoproterenol no longer produced a significant increase in extracellular adenosine accumulation (Fig. 7). In these experiments, in the presence of IBMX alone, isoproterenol produced a $56 \%$ increase in extracellular adenosine accumulation $(p<0.05)$. In contrast, with the addition of $180 \mu \mathrm{M}$ RO 20-1724, there was no significant change in extracellular adenosine concentration with isoproterenol.

We also demonstrated the effect of the combination of IBMX with RO 20-1724 on the time course of extracellular adenosine as well as cAMP accumulation (seven experiments performed). As expected from the data presented in the previous figure, isoproterenol-stimulated adenosine accumulation was significantly inhibited by RO 20-1724 plus IBMX at 30 min ( $p<$ 0.01 ) (Fig. 8A). Although there have been reports of phosphodiesterase inhibitors blocking cAMP secretion (King and Mayer, 1974; Wu et al., 1978; Nemecek et al., 1980), we found that IBMX plus RO 20-1724, at the doses used, did not block cAMP
$A$
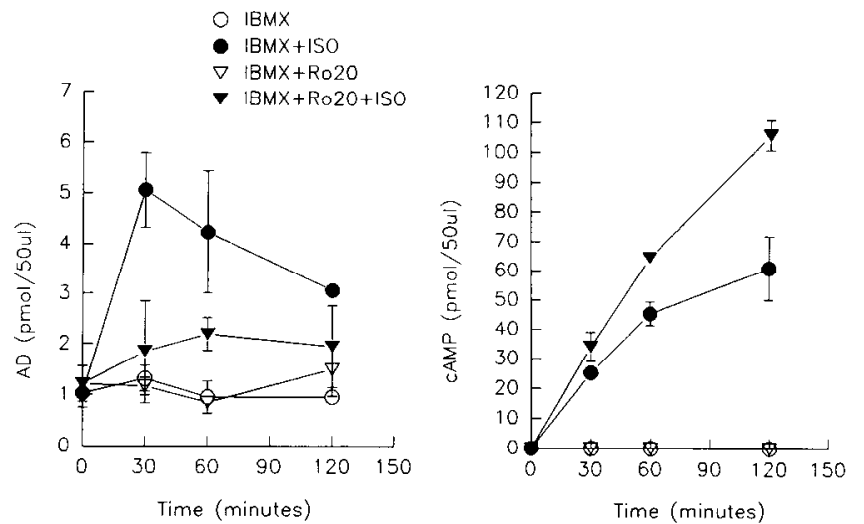

Figure 8. Effect of IBMX plus RO 20-1724 on the time course of isoproterenol-stimulated adenosine and cAMP accumulation. Astrocyte-rich cultures were preincubated with $100 \mu \mathrm{M}$ IBMX alone, or IBMX plus $180 \mu \mathrm{M}$ RO 20-1724. At zero time, $1 \mu \mathrm{M}$ isoproterenol or vehicle was added to cultures in each of these groups, and medium was collected at selected intervals for assay of adenosine and cAMP. Isoproterenol evoked a significant increase in extracellular adenosine in this experiment, in the presence of $100 \mu \mathrm{M}$ IBMX alone, which peaked at $30 \mathrm{~min}$. RO 20-1724 plus IBMX inhibited the accumulation of adenosine $(p<$ 0.01 at $30 \mathrm{~min}$ ). In $B$, the effect of isoproterenol on extracellular cAMP accumulation in the presence of IBMX and in the presence of IBMX plus RO 20-1724 is shown. cAMP measured in the medium was significantly increased by the addition of RO 20-1724 to IBMX at $60 \mathrm{~min}$ $(p<0.05)$ and $120 \mathrm{~min}(p<0.001)$. Data shown are from a single experiment of seven that were performed.

secretion and, in fact, that the extracellular cAMP was equal to or greater than control levels stimulated by isoproterenol, as seen in this representative single experiment (Fig. $8 B$ ). Isoproterenol-stimulated extracellular cAMP accumulation was significantly greater in the presence of RO 20-1724 than in its absence at $60(\mathrm{~min} p<0.05)$ and at $120 \mathrm{~min}(p<0.001)$.

We have tried using representatives of other classes of phosphodiesterase inhibitors in conjunction with $100 \mu \mathrm{M}$ IBMX, such as dipyridamolc, carbazcran, and trifluoperazine (all at 10 $\mu \mathrm{M})$, but found no blockade of isoproterenol-stimulated extracellular adenosine accumulation. Higher concentrations of inhibitors were not used in these experiments because they were found to have large effects (greater than a doubling) on adenosine levels themselves.

\section{Effect of probenecid on isoproterenol-stimulated extracellular cAMP and adenosine accumulation}

The pharmacology of inhibition of cAMP efflux has been well characterized in a number of cell types, including astrocytomaderived cell lines (Doore et al., 1975; Henderson and Strauss, 1991). If extracellular adenosine that appears as a result of $\beta$-adrenergic receptor stimulation is derived from extracellular cAMP, then drugs such as probenecid, which block cAMP efflux, should eliminate this effect of isoproterenol. The effect of probenecid on isoproterenol-stimulated adenosine accumulation is shown in Figure 9, which represents data pooled from 10 experiments. In these experiments, the adenosine concentration in medium from isoproterenol-treated cultures was $177 \pm 66 \%$ of control values, and this effect was blocked by $1000 \mu \mathrm{M}$ probenecid ( $p$ $<0.05$ ). [In these experiments the baseline adenosine concentration was $1.8 \pm 1.2 \mathrm{pmol} / 50 \mu \mathrm{l}$ without probenecid and 2.0 


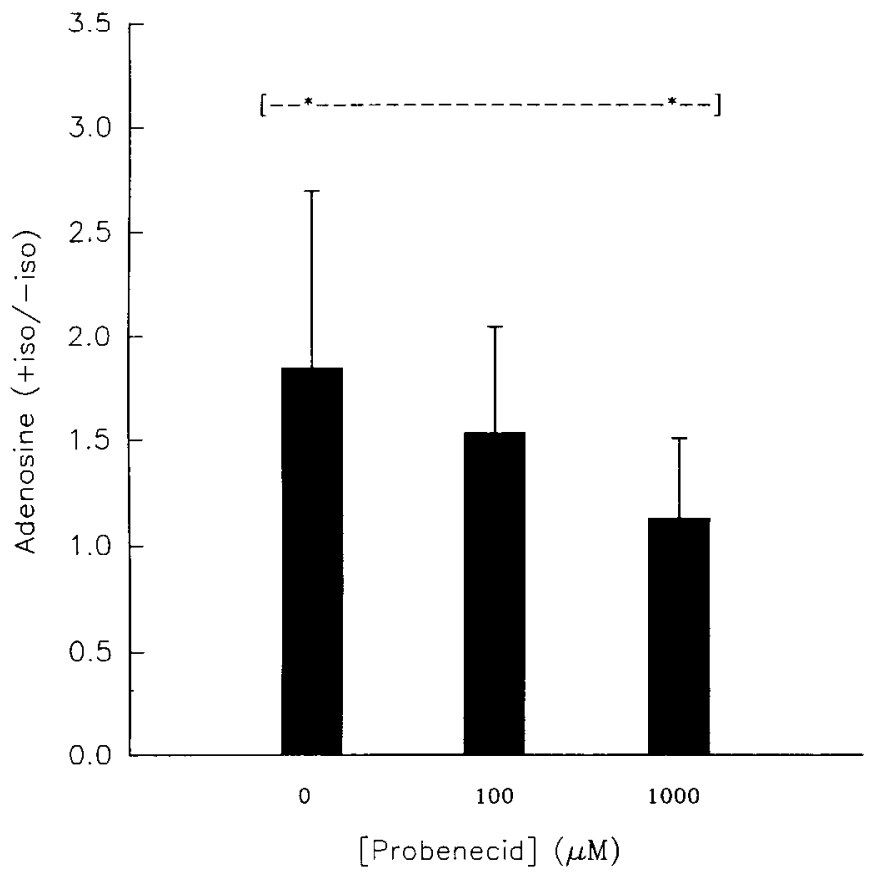

Figure 9. Efrect of probenecid on isoproterenol-stimulated adenosine accumulation. Probenecid blocks isoproterenol-stimulated adenosine accumulation in astrocyte-rich cultures. For each concentration of probenecid the ratio of the adenosine concentration with isoproterenol stimulation over the adenosine concentration without isoproterenol stimulation was obtained. The results from 10 separate experiments were pooled. There was a significant difference $(p<0.05)$ between the values obtained in the absence of probenecid and in the presence of $1000 \mu \mathrm{M}$ prohenecid (populations heing compared indicated by asterisks embedded in dashed line). Astrocyte-rich cultures were exposed to 0 , 100 , and $1000 \mu \mathrm{M}$ probenecid in the presence or absence of $1 \mu \mathrm{M}$ isoproterenol for $30 \mathrm{~min}$. Media samples were then taken and assayed for adenosine, shown here, and cAMP (shown in Fig. 10). These data show the pooled results from 10 similar experiments. In four of these experiments, $100 \mu \mathrm{M}$ IBMX was present in the medium, but there were no significant differences between the results from these experiments with IBMX and experiments performed without IBMX, and therefore they were pooled.

$\pm 1.4 \mathrm{pmol} / 50 \mu \mathrm{l}$ with probenecid $(1000 \mu \mathrm{M})$.] This concentration of probenecid also decreased extracellular cAMP by $78 \%$ in these experiments $(p<0.01)$ (Fig. 10).

\section{Discussion}

We have shown that cortical cultures respond to stimulation by the $\beta$-adrencrgic agonist isoproterenol by secreting cAMP into the extracellular medium. This response can be detected at concentrations as low as $10 \mathrm{nM}$, and saturates by $1 \mu \mathrm{M}$. We noticed in some experiments what appeared to be a concentration dependence of the time for the extracellular concentration of cAMP to attain a maximum, as seen in Figure 1, and assume that this is due to a change in the relative contributions of the source of extracellular CAMP, cAMP transport, and the sink for cAMP, extracellular cyclic nucleotide phosphodiesterase activity, at different isoproterenol concentrations.

Using the peak concentrations of extracellular cAMP at different concentrations of isoproterenol, we obtained a value for the $\mathrm{EC}_{50}$ for the stimulation of cAMP transport by isoproterenol of approximately $100 \mathrm{nM}$ (Fig. 2). By comparison, in other work using glial cultures and investigating the activation by isoproterenol of adenylyl cyclase by measuring intracellular cAMP,

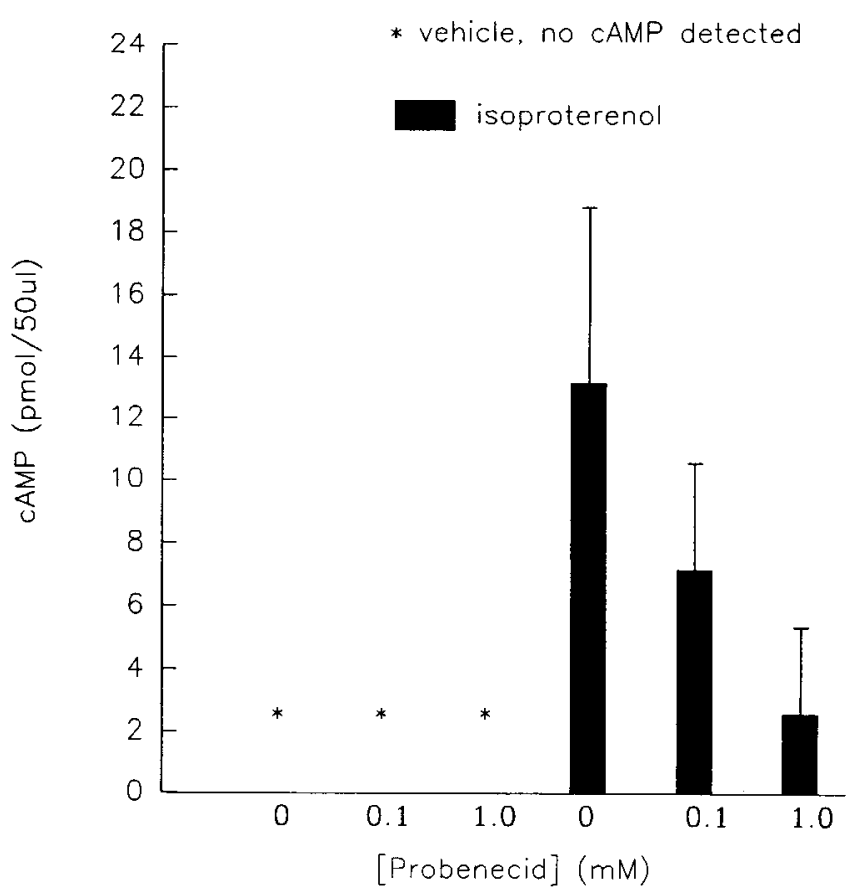

Figure 10. Effect of probenecid on isoproterenol-stimulated cAMP accumulation. Probenecid blocks isoproterenol-stimulated cAMP secretion. This figure shows pooled data from six experiments, in which probenecid is shown to have a dose-dependent effect on cAMP secretion. The $\mathrm{IC}_{50}$ for the inhibition of cAMP secretion by probenecid in these experiments was $89 \pm 6 \mu \mathrm{M}$. No cAMP was detectable in the medium without isoproterenol stimulation (shown by asterisks).

an $\mathrm{EC}_{50}$ of $43 \mathrm{nM}$ was determined (Ebersolt et al., 1981). A number of factors might account for the difference between the value we have determined and this value, including the fact that we performed our experiments on mixed cultures of neurons and glial cells, whereas the previous experiments were performed on glial cultures, or that in our experiments we measured extracellular cAMP whereas in the previous experiments intracellular cAMP was assayed.

The principal question that we have examined in this study is whether $\beta$-adrenergic receptor stimulation might cause an increase in extracellular adenosine derived from extracellular cAMP. In fact, we were able to demonstrate, using an HPLC method, that stimulation of astrocyte-rich cultures with isoproterenol resulted in an increase in extracellular adenosine. This effect was not observed in every experiment, but in a large series of cxpcrimcnts, wc found a highly significant increase in extracellular adenosine in cultures stimulated with isoproterenol. Our experiments comparing astrocyte-rich cultures, neuron-enriched astrocyte-poor cultures, and astrocyte cultures suggest one explanation why this might be the case. The fact that isoproterenol produces an increase in cAMP in both astrocyte cultures as well as astrocyte-rich cultures, but an increase in adenosine only in mixed cultures of neurons and astrocytes (astrocyte-rich cultures), implies that one or both of the enzymes required for the breakdown of cAMP occurs in neurons. Since we know that astrocytes (in addition to neurons) (Nagy et al., 1986; Trapido-Rosenthal et al., 1990) possess abundant 5'-nucleotidase (Kreutzberg et al., 1978), it seems likely that extracellular cyclic nucleotide phosphodiesterase activity is localized to neurons in the cultures. We do not know whether this enzyme is present on all neurons or only on a subpopulation of neurons, 
or what is the subcellular localization of this enzyme on neurons that possess it. In any case, this apparent requirement for neurons in order to detect isoproterenol-stimulated adenosine accumulation suggests that there might be a minimum density of neurons below which an insufficient amount of enzyme would be present to produce a detectable amount of adenosine derived from cAMP. Because it is possible that extracellular cyclic nucleotide phosphodiesterase is present not on all cortical neurons but on a subpopulation, it is conceivable that that subpopulation is variably represented in different cultures. This might also account for the variability in the response to isoproterenol. Finally, the fact that no effect of probenecid is seen below $100 \mu \mathrm{M}$ suggests that the phosphodiesterase is probably saturated at low levels of cAMP and, as a result, no effect of suppressing cAMP transport is seen until extracellular cAMP concentrations are markedly reduced. This observation suggests that the absolute amount of enzyme might be limiting, and therefore a major factor in determining whether an effect of isoproterenol is observable in these cultures. Another issue that might bear on the reproducibility of this phenomenon is that these cultures are not a monolayer. They consist instead of a sandwich of neuropil with an astrocyte layer both underneath, in contact with the substrate, and on top, sequestering the neuropil from the extracellular medium (Harris and Roscnberg, 1993). Therefore, cAMP secreted from the surface of astrocytes may go into the medium without ever coming into contact with cyclic nucleotide phosphodiesterase associated with neurons. cAMP secreted by astrocytes that are in contact with the neuropil, on the other hand, may come in contact with a neuronal extracellular enzyme, but because of the tortuous route to the surface, which might be occluded by tight junctions (Harris and Rosenberg, 1993), may never gain access to the extracellular medium where it could be detected. Therefore, the fact that our sampling is restricted to the extracellular medium may limit our ability to detect the conversion of cAMP to a metabolite if this conversion were to depend on an interaction with dendrites. Nonetheless, we have been able to demonstrate an effect of isoproterenol in vitro, and this provides strong motivation to pursue these studies in vivo, using microdialysis techniques and sampling directly in the extraccllular space. Using these techniques it has already been demonstrated that $\beta$-adrenergic stimulation produces secretion of cAMP in vivo, in the frontal cortex of living rats (Stone and John, 1990).

The demonstration that $\beta$-adrenergic stimulation of cortical cultures increases extracellular adenosine still leaves open the question of whether the adenosine that accumulates in the extracellular space actually derived from extracellular cAMP. If this were true, then it should be possible to block this effect of isoproterenol both with an inhibitor of cyclic nucleotide phosphodiesterase and with an inhibitor of cAMP transport. The usefulness of a number of phosphodiesterase inhibitors tested was limited by their tendency to increase baseline levels of adenosine, in the absence of $\beta$-adrenergic receptor stimulation. We do not understand the basis for this effect, but it may be due to activity of these agents as adenosine receptor antagonists (Bruns and et al., 1986; Parsons et al., 1988; Williams and Jarvis, 1988; Nicholson et al., 1989). We used concentrations of these drugs that did not on their own increase adenosine concentrations by more than $100 \%$, and with these constraints, found no effect of a variety of phosphodiesterase inhibitors, including the nonspecific inhibitor IBMX (Fig. 7). This apparent resistance to the effect of IBMX is unusual, though not unprecedented (Lavan et al., 1989). However, we found that the combination of RO 201724 with IBMX did block isoproterenol-stimulated adenosine accumulation (Figs. 7, 8) and, as expected, increased cAMP accumulation (Fig. 8). The exact relationship between extracellular adenosine levels and extracellular cAMP levels in these experiments is complicated by a number of factors, including the effects of the phosphodiesterase inhibitors on the intracellular lcvel of cAMP (which detcrmines the rate of cAMP transport) (Barber and Butcher, 1983) and adenosine uptake. For this reason, one would not necessarily expect a stoichiometric relationship between the decrease in the adenosine and the increase in the cAMP detected in the medium. Nonetheless, the data support the hypothesis that cAMP is a source of the adenosine, since a decrease in adenosine is associated with an increase in cAMP. In the experiment shown in Figure 8, a decrease in adenosine content of $3.2 \mathrm{pmol} / 50 \mu \mathrm{l}$ was associated with an increase in cAMP content of $8.9 \mathrm{pmol} / 50 \mu \mathrm{l}$.

The significance of the requirement for two different phosphodiesterase inhibitors is unclear. Previous studies have demonstrated a subtype of cyclic nucleotide phosphodiesterase that has both regulatory and catalytic cyclic nucleotide binding sites that interact (Francis et al., 1990; Thomas et al., 1992). Therefore, it is conceivable that the interaction of IBMX with the enzyme we are studying changes the affinity of the catalytic site for RO 20-1724, or vice versa. Clearly, more detailed pharmacological studies need to be performed in order to characterize the extracellular phosphodiesterase activity that we observe in cortical cultures. These studies would best be performed in a simple assay of phosphodiesterase activity, rather than in the experimental paradigm we have employed here, which demonstrates phosphodiesterase activity indirectly.

As another test of the hypothesis that the adenosine that accumulates in response to isoproterenol stimulation derives from extracellular cAMP, we attempted to block the effect of isoproterenol by using probenecid, an inhibitor of cAMP transport. We found an $\mathrm{EC}_{50}$ for the inhibition by probenecid of cAMP secretion of $89 \pm 6 \mu \mathrm{M}$ (Fig. 10). This is similar to the value obtained previously by others for the effect of probenecid on cAMP extrusion from C6 rat glioma cells $(83 \pm 17 \mu \mathrm{M})$ (Henderson and Strauss, 1991). In contrast, with respect to the potency of probenecid as an inhibitor of isoproterenol-stimulated adenosine accumulation, we found little effect below 100 $\mu \mathrm{M}$. In the experiments shown in Figure 9, the $\mathrm{EC}_{50}$ appears to be between 200 and $300 \mu \mathrm{M}$. These data are consistent with the hypothesis that the origin of the adenosine that accumulates in response to isoproterenol is extracellular cAMP, but also suggest that cAMP sccrction itsclf is not the ratc-limiting step.

Until recently, no studies have examined the possibility that hydrolysis of extracellular cAMP might provide a significant source of extracellular adenosine in cerebral cortex. In spinal cord, serotonin $(5-\mathrm{HT})$ has been shown to release a nucleotide from dorsal horn spinal cord synaptosomes that is degraded extracellularly to adenosine, leading to the hypothesis that this mechanism might in part underlie the antinociception produced by 5 -HT (DeLander and Hopkins, 1987; Sweeney et al., 1988; Sawynok and Sweeney, 1989). Sweeney et al. (1990) present evidence that this nucleotide is, in fact, cAMP. Interestingly, in the dorsal horn synaptosomes, purine release is $\mathrm{Ca}^{2+}$ dependent, originates in capsaicin-sensitive small-diameter primary afferent nerve terminals (Sweeney et al., 1988), and is not elicited by noradrenergic stimulation. Therefore, the source of extracellular cAMP in the spinal cord appears to be neurons, and 
c $\triangle M P$ in this case appears to be transported by a calciumdependent process - possibly exocytosis. In contrast, in the process we are suggesting as a source of adenosine in cortex, extracellular cAMP derives from astrocytes (Rosenberg and Dichter, 1989), secreted in response to norepinephrine via a transport process that is ATP dependent (Barber and Butcher, 1983; Henderson and Strauss, 1991) and calcium independent (Barber and Butcher, 1981).

cAMP efflux occurs in all cells that accumulate cAMP intracellularly in response to $\beta$-adrenergic stimulation (Barber and Butcher, 1983), and was originally discovered by Davoren and Sutherland (1963) in the pigeon erythrocyte. This transport process has been studied in several different preparations, and has been demonstrated in the frontal cortex of living rats (Egawa et al., 1988), in the striatal slice preparation (Schoffelmeer et al., 1985; Headley and O'Shaughnessy, 1986; O'Shaughnessy et al., 1987), in glioma-derived cell lines (Doore et al., 1975; Henderson and Strauss, 1991), and in rat cerebral cortex in culture (Rosenberg and Dichter, 1989). The transport mechanism is characteristically inhibited by several structurally diverse compounds including probenecid, verapamil, dipyridamole, and prostaglandin Al, and this pharmacology is similar to that of the multidrug resistance transporter, with which it appears to bc closcly related (Henderson and Strauss, 1991). It is noteworthy that the structure of adenylyl cyclase itself is similar to that of the multidrug resistance transporter, leading to the suggestion that the adenylyl cyclase molecule provides an efflux route for cAMP (Krupinski et al., 1989). The function of cAMP efflux has been a subject of speculation since its discovery, but, in any case, this transport route appears to provide a pathway of emux for large organic ions from cells in which it appears, as is the case for the multidrug resistance transporter or P-glycoprotein. The possible functional significance of cAMP efflux has been previously reviewed (Barber and Butcher, 1983; Rosenberg, 1993). Recently, a new addition has been made to the list of possibilities with the demonstration that P-glycoprotein is itself or is capable of regulating a volume-sensitive chloride conductance (Valverde et al., 1992). In addition, Sorbera and Morad (1991) have shown that cAMP diminishes sodium currents in myocytes, suggesting the existence of an extracellular cAMP receptor on this cell type. This phenomenon has not yet been described in neurons.

Although there is ample evidence for the presence of $\beta$-adrenergic receptors on neurons in the adult cerebral cortex and hippocampus (Madison and Nicoll, 1986a,b; Aoki et al., 1987; Schwindt et al., 1988; Aoki and Pickel, 1990, 1992), there is very strong evidence as well that astrocytes are a major target for the $\beta$-adrenergic actions of norepinephrine (Stone and Ariano, 1989; McCarthy et al., 1991). In fact, in embryonic cultures of cerebral cortex there is compelling evidence that cortical neurons do not have $\beta$-adrenergic receptors (Rosenberg and Dichter, 1989; McCarthy et al., 1991). What role might $\beta$-adrenergic stimulation of adenosine accumulation play in the physiology of norepinephrine? Since Woodward and colleagues proposed that the function of the norepinephrine projection to cortex is to increase the signal-to-noise relationship for significant stimuli, this hypothesis has become widely accepted as useful in understanding the function of norepinephrine (Woodward et al., 1979; Foote et al., 1983). It has received considerable support in studies (Haas and Konnerth, 1982; Madison and Nicoll, 1982) that provided a cellular basis for the strengthening of strong inputs that is one role for norepinephrine required by the Wood- ward hypothesis. However, another function for norepinephrine posited by the Woodward hypothesis is the suppression of weak inputs. Madison and Nicoll showed that norepinephrine causes a small hyperpolarization associated with an increase in membrane conductance in approximately $70 \%$ of pyramidal neurons in the CAl region of hippocampus, and demonstrated that this was sufficient to inhibit action potential generation in response to a near-threshold stimulus (Madison and Nicoll, 1986a). Another, possibly additional, mechanism for the suppression of weak inputs suggested by the present work is that by increasing extracellular adenosine concentration, excitatory synaptic inputs might be effectively weakened by decreasing excitatory transmitter release.

An unresolved issue is determining in what time frame this effect might occur. The phenomenon we have demonstrated in culture is slow, occurring over many minutes, whereas one would expect an effective physiological mechanism to occur over seconds or fractions of a second. However, our sampling of the extracellular medium is at best an imperfect window onto events that are taking place next to synapses that are surrounded by other cells, including astrocytes, from which they are separated by very small volumes. In these small volumes, rapid changes in the concentration of solutes is possible with the transport of small quantities of substrates across cell membranes of either neurons or adjacent glial cells. Although tissue culture provides an extremely useful model system in which it is possible to apply drugs at known concentrations, to measure their effects on cells, and to study the role of cellular interactions in generating these effects, ultimately it will be necessary to investigate the phenomenon we have described in vivo using microdialysis techniques. In addition, once the components of the system have been elucidated, as well as how to manipulate them pharmacologically, it would be of great interest to use this information to study the role of the mechanism we have described in a variety of electrophysiological model systems.

In addition to monoamines, the cortex is richly endowed with neuropeptides, some of which are coupled to adenylyl cyclase. Any hormone coupled to adenylyl cyclase (positively or negatively) might be expected to modulate cAMP efflux. Therefore, a mechanism deriving adenosine from cAMP might be a final common pathway by which multiple neuromodulators could converge to control extracellular adenosine, and thereby inhibitory tone. Such a mechanism might have important effects in modulating synaptic transmission, both under normal circumstances and under the abnormal circumstances of hypoxia/ischemia or seizures (Dragunow et al., 1985; Dragunow and Robcrtson, 1987; Dragunow, 1988; Dragunow and Faull, 1988).

\section{References}

Aoki C, Pickel VM (1990) Ultrastructural immunocytochemical evidence for presynaptic localization of beta-adrenergic receptors in the striatum and cerebral cortex of rat brain. Ann NY Acad Sci 604:582585.

Aoki C, Pickel VM (1992) Ultrastructural relations between betaadrencrgic receptors and catecholaminergic ncurons. Brain Res Bull 29:257-263.

Aoki C, Milner TA, Sheu K-FR, Blass JP, Pickel VM (1987) Ultrastructural localization of beta-adrenergic receptor-like immunoreactivity in the rat cortex and neostriatum of rat brain. Brain Res 437: 264-282.

Barber R, Butcher RW (1981) The quantitative relationship between intracellular concentration and egress of cyclic AMP from cultured cells. Mol Pharmacol 19:38-43.

Barber R, Butcher RW (1983) The egress of cyclic AMP from metazoan cells. Adv Cyclic Nucleotide Res 15:119-138. 
Beavo J (1988) Multiple isozymes of cyclic nucleotide phosphodiesterase. In: Advances in second messenger and phosphoprotein research, Vol 22 (Greengard P, Robison GA, eds), pp 1-38. New York: Raven.

Beavo JA, Reifsnyder DH (1990) Primary sequence of cyclic nucleotide phosphodiesterase isozymes and the design of selective inhibitors. Trends Pharmacol Sci 11:150-155.

Bruns RF, Daly JW, Snyder SH (1980) Adenosine receptors in brain membranes: binding of $\mathrm{N}^{6}$-cyclohexyl[ $\left.{ }^{3} \mathrm{H}\right]$ adenosine and 1,3-diethyl8-[3]phenylxanthine. Proc Natl Acad Sci USA 77:5547-5551.

Bruns RF et al. (1986) Characterization of the A2 adenosine receptor labelled by $\left[{ }^{3} \mathrm{H}\right]-\mathrm{NECA}$ in rat striatal membranes. Mol Pharmacol 29: 331.

Burke SP, Nadler JV (1988) Regulation of glutamate and aspartate release from slices of the hippocampal CA1 area: effects of adenosine and baclofen. J Neurochem 51:1541-1551.

Challiss RAJ, Nicholson CD (1990) Effects of selective phosphodiesterase inhibition on cyclic AMP hydrolysis in rat ccrebral cortical slices. Br J Pharmacol 99:47-53.

Choi DW (1987) Ionic dependence of glutamate neurotoxicity. J Neurosci 7:369-379.

Corradetti R, LoConte G, Moroni F, Passani MB, Pepeu G (1984) Adenosine decreases aspartate and glutamate release from hippocampal slices. Eur J Pharmacol 104:19-26.

Davoren PR, Sutherland EW (1963) The effect of $l$-epinephrine and other agents on the synthesis and release of adenosine $3^{\prime}, 5^{\prime}$-phosphate by whole pigeon erythrocytes. J Biol Chem 238:3009-3015.

DeLander GE, Hopkins CJ (1987) Interdependence of spinal adenosinergic, serotonergic and noradrenergic systems mediating antinociception. Neuropharmacology 26:1791-1794.

Dolphin AC, Archer ER (1983) An adenosine agonist inhibits and a cyclic AMP analogue enhances the releasc of glutamate but not GABA from slices of rat dentate gyrus. Neurosci Lett 43:49-54.

Dolphin AC, Forda SR, Scott RH (1986) Calcium-dependent currents in cultured rat dorsal root ganglion neurones are inhibited by an adenosine analogue. J Physiol (Lond) 373:47-61.

Doore BJ, Bashor MM, Spitzer N, Mawe RC, Saier MH (1975) Regulation of adenosine $3^{\prime}: 5^{\prime}$-monophosphate efflux from rat glioma cells in culture. J Biol Chem 250:4371-4372.

Douglas WW, Rubin RP (1963) The mechanism of catecholamine release from adrenal medulla and the role of calcium in stimulussecretion coupling. J Physiol (Lond) 167:288-310.

Dragunow M (1988) Purinergic mechanisms in epilepsy. Prog Neurobiol 31:85-108.

Dragunow M, Faull RLM (1988) Neuroprotective effects of adenosine. Trends Pharmacol Sci 9:193-194.

Dragunow M, Robertson HA (1987) 8-Cyclopentyl 1,3-dimethylxanthine prolongs epileptic seizures in rats. Brain Res 417:377-379.

Dragunow M, Goddard GV, Laverty R (1985) Is adenosine an enogenous anticonvulsant? Epilepsia 26:480-487.

Dunwiddie TV (1984) Interactions between the effects of adenosine and calcium on synaptic responses in rat hippocampus in vitro. $J$ Physiol (Lond) 350:545-559.

Dunwiddie TV (1985) The physiological role of adenosine in the central nervous system. Int Rev Neurobiol 27:63-139.

Ebersolt C, Perez M, Vassent G, Bockaert J (1981) Characteristics of the beta1- and beta2-adrenergic-sensitive adenylate cyclases in glial cell primary cultures and their comparison with beta2-adrenergicsensitive adenylate cyclase of meningeal cells. Brain Res 213:151161.

Egawa M, Hoebel BG, Stone EA (1988) Use of microdialysis to measure brain noradrenergic receptor function in vivo. Brain Res 458: 303-308.

Erecinska M, Dagani F (1990) Relationships between the neuronal sodium/potassium pump and energy metabolism. J Gen Physiol 95: 591-616.

Fastbom J, Fredholm BB (1985) Inhibition of $\left[{ }^{3} \mathrm{H}\right]$ glutamate release from rat hippocampal slices by L-phenylisopropyladenosine. Acta Physiol Scand 125:121-123.

Foote SL, Bloom FE, Aston-Jones G (1983) Nucleus locus coeruleus: new evidence of anatomical and physiological specificity. Physiol Rev 63:844-914.

Francis SH, Thomas MK, Cirbin JD (1990) Cyclic GMP-binding cyclic GMP-specific phosphodiesterase from lung. In: Cyclic nucleotide phosphodiesterases: structure, regulation, and drug action (Beavo J, Houslay MD, eds), pp 117-140. Chichester: Wiley.
Fredholm BB, Dunwiddie TV (1988) How does adenosine inhihit transmitter release? Trends Pharmacol Sci 9:130-134.

Goodman RR, Kuhar MJ, Hester L, Snyder S (1983) Adenosine receptors: autoradiographic evidence for their location on axon terminals of excitatory neurons. Science 220:967-969.

Haas HL, Konnerth A (1982) Histamine and noradrenaline decrease calcium-activated potassium conductance in hippocampal pyramidal cells. Nature 302:432-434.

Harris KM, Rosenberg PA (1993) Localization of synapses in rat cerebral cortex in dissociated cell culture. Neuroscience 53:495-508.

Headley PM, O'Shaughnessy CT (1986) The use of cyclic AMP efflux studies in attempts to determine the effects of morphine on cyclic AMP formation in striatal slices. Neuropharmacology 25:919-922.

Henderson GB, Strauss BP (1991) Evidence for cAMP and cholate extrusion in $\mathrm{C} 6$ rat glioma cells by a common anion efflux pump. J Biol Chem 266:1641-1645.

Hoehn K, White TD (1990a) Role of excitatory amino acid receptors in $\mathrm{K}^{+}$- and glutamate-evoked release of endogenous adenosine from rat cortical slices. J Neurochem 54:256-265.

Hoehn K, White TD (1990b) Glutamate-evoked release of endogenous adenosine from ral cortical synaptosomes is mediated by glutamate uptake and not by receptors. J Neurochem 54:1716-1724.

Hoehn K, White TD (1990c) $N$-methyl-D-aspartate, kainate, and quisqualate release endogenous adenosine from rat cortical slices. Neuroscience 39:441-450.

King CD, Mayer SE (1974) Inhibition of egress of adenosine 3', 5'monophosphate from pigeon erythrocytes. Mol Pharmacol 10:941953.

Kreutzherg GW, Barron KD, Schubert P (1978) Cytochemical localization of $5^{\prime}$-nucleotidase in glial plasma membranes. Brain Res 158: 247-257.

Krupinski J, Coussen F, Bakalyar HA, Tang W-J, Feinstein PG, Orth K. Slaughter C, Reed RR, Gilman AG (1989) Adenylyl cyclase amino acid sequence: possible channel- or transporter-like structure. Science 244:1558-1564.

Lavan BE, Lakey T, Houslay MD (1989) Resolution of soluble cyclic nucleotide phosphodiesterase isoenzymes, from liver and hepatocytes, identifies a novel IBMX-insensitive form. Biochem Pharmacol 38:4123-4136.

Levin RM, Weiss B (1977) Binding of trifluoperazine to the calciumdependent activator of cyclic nucleotide phosphodiesterase. Mol Pharmacol 13:690-697.

MacDonald RL, Skerritt JH, Werz MA (1986) Adenosine agonists reduce voltage-dependent calcium conductance of mouse sensory neurones in culture. J Physiol (Lond) 370:75-90.

MacDonald WF, White TD (1985) Nature of extrasynaptosomal accumulation of endogenous adenosine evoked by $\mathrm{K}^{+}$and veratridine. J Neurochem 45:791-797.

Madison DL, Fox AP, Tsien RW (1987) Adenosine reduces an inactivating component of calcium current in hippocampal CA3 neurons. Biophys $\mathrm{J}$ 51:30a.

Madison DV, Nicoll RA (1982) Noradrenaline blocks accommodation of pyramidal cell discharge in the hippocampus. Nature 299:636-638.

Madison DV, Nicoll RA (1986a) Actions of noradrenaline recorded intracellularly in rat hippocampal CA1 pyramidal neurones, in vitro. J Physiol (Lond) 372:221-244.

Madison DV, Nicoll RA (1986b) Cyclic adenosine 3',5'-monophosphate mediates beta-receptor actions of noradrenaline in rat hippocampal pyramidal cells. J Physiol (Lond) 372:245-259.

McCarthy KD, Salm A, Lerea LS (1991) Astroglial receptors and their regulation of intermediate filament protein phosphorylation. In: Glial cell receptors (Kimelberg HK, ed), pp 1-22. New York: Raven.

Meghji P, Tuttle JB, Rubio R (1989) Adenosine formation and releasc by embryonic chick neurons and glia in cell culture. J Neurochem 53:1852-1860.

Nagy AK, Shuster TA, Delgado-Escueta AV (1986) Ecto-ATPase of mammalian synaptosomes: identification and enzymic characterization. J Neurochem 47:976-986.

Nemecek GM, Wells JN, Butcher RW (1980) Inhibition of fibroblast cyclic AMP escape and cyclic nucleotide phosphodiesterase activities by xanthines. Mol Pharmacol 18:57-64.

Nicholson CD, Jackman SA, Wilke R (1989) The ability of denbufylline to inhibit cyclic nucleotide phosphodiesterase and its affinity for adenosine receptors and the re-uptake site. $\mathrm{Br} \mathrm{J}$ Pharmacol 97: 889-897.

Nicholson CD, Chaliss RAJ, Shahid M (1991) Differential modulation 
of tissue function and therapeutic potential of selective inhibitors of cyclic nucleotide phosphodiesterase isoenzymes. Trends Pharmacol Sci 12:19-27.

Okada Y, Ozawa S (1991) Inhibitory action of adenosine on synaptic transmission in the hippocampus of the guinea pig in vitro. Eur $\mathbf{J}$ Pharmacol 68:483-492.

O'Shaughnessy CT, Poat JA, Turnbull MJ (1987) Cyclic AMP efflux from rat striatal slices is enhanced by CCK. Biochem Pharmacol 36: 976-979.

Paes de Carvalho R, Braas KM, Snyder SH, Adler R (1990) Analysis of adenosine immunoreactivity, uptake, and release in purified cultures of developing chick embryo retinal neurons and photoreceptors. J Neurochem 55:1603-1611.

Parsons WJ, Ramkumar V, Stiles GL (1988) The new cardiotonic agent sulmazole is an $\mathrm{A} 1$ adenosine receptor antagonist and functionally blocks the inhibitory regulator, Gi. Mol Pharmacol 33:441448.

Perrett D (1987) Determination of adenosine ribo- and deoxyribonucleotides as their 1- $\mathrm{N}^{6}$-etheno derivatives by reversed-phase ionpair high performance liquid chromatography. J Chromatogr 386: 289-296.

Philibert RA, Dutton GR (1989) Dihydropyridines modulate $\mathrm{K}^{+}$evoked amino acid and adenosine release from cerebellar neuronal cultures. Neurosci Lett 102:97-102.

Plagemann PGW, Wohlhueter RM (1980) Permeation of nucleosides, nucleic acid basis and nucleotides in animal cells. Curr Top Membr Transp 14:225-330.

Proctor WR, Dunwiddie TV (1983) Adenosine inhibits calcium spikes in hippocampal pyramidal neurons in vitro. Neurosci Lett 35:197201.

Ramnath RR, Strange K, Rosenberg PA (1992) Neuronal injury evoked by depolarizing agents in rat cortical cultures. Neuroscience, in press.

Rosenberg PA (1991) Accumulation of extracellular glutamate and neuronal death in astrocyte-poor cortical cultures exposed to glutamine. Glia 4:91-100.

Rosenberg PA (1993) Functional significance of cyclic AMP secretion in cerebral cortex. Brain Res Bull 29:315-317.

Rosenberg PA, Aizenman E (1989) Hundred-fold increase in neuronal vulnerability to glutamate toxicity in astrocyte-poor cultures of rat cerebral cortex. Neurosci Lett 103:162-168.

Rosenberg PA, Dichter MA (1989) Extracellular cAMP accumulation and degradation in rat cerebral cortex in dissociated cell culture. $J$ Neurosci 9:2654-2663.

Sawynok J, Sweeney MI (1989) Role of purines in nociception. Neuroscience 32:557-569.

Schoffelmeer ANM, Hansen HA, Stoof JC, Mulder AH (1985) Inhibition of dopamine-stimulated cyclic AMP efflux from rat neostriatal slices by activation of mu and delta opioid receptors: a permissive role for D-2 dopamine receptors. Eur J Pharmacol 118:363-366.

Scholz KP, Miller RJ (1991) Analysis of adenosine actions on calcium currents and synaptic transmission in cultured pyramidal neurons. $J$ Physiol (Lond) 435:373-393.

Schubert P, Mitzdorf U (1979) Analysis and quantitative evaluation of the depressive effect of adenosine on evoked potentials in hippocampal slices. Brain Res 172:186-190.

Schwindt PC, Spain WJ, Foehring RC, Chubb MC, Crill WE (1988) Slow conductances in neurons from cat sensorimotor cortex in vitro and their role in slow excitability changes. $J$ Neurophysiol 59:450467.

Secrist JA, Barrio JR, Leonard NJ, Weber G (1972) Fluorescent modification of adenosine-containing coenzymes. Biological activities and spectroscopic properties. Biochemistry 11:3499-3506.

Sorbera LA, Morad M (1991) Modulation of cardiac sodium channels by cAMP receptors on the myocyte surface. Science 253:1286-1289.

Stone EA, Ariano MA (1989) Are glial cells targets of the central noradrenergic system? A review of the evidence. Brain Res Rev 14: 297-309.

Stone EA, John SM (1990) In vivo measurement of extracellular cyclic AMP in the brain: use in studies of beta-adrenoceptor function in nonanesthetized rats. J Neurochem 55:1942-1949.

Sweeney MI, White TD, Sawynok J (1988) 5-Hydroxytryptamine reIcases adenosine from primary afferent nerve terminals in the spinal cord. Brain Res 462:346-349.

Sweeney MI, White TD, Sawynok J (1990) 5-Hydroxytryptamine releases adenosine and cyclic AMP from primary afferent nerve terminals in the spinal cord in vivo. Brain Res 528:55-61.

Thomas MK, Francis SH, Beebe SJ, Gettys TW, Corbin JD (1992) Partial mapping of cyclic nucleotide sites and studies of regulatory mechanisms of phosphodiesterases using cyclic nucleotide analogues. In: The biology of cyclic nucleotide phosphodiesterases (Strada SJ, Hidaka H, eds), pp 45-54. New York: Raven.

Trapido-Rosenthal HG, Carr WES, Gleeson RA (1990) Ectonucleotidase activities associated with the olfactory organ of the spiny lobster. J Neurochem 55:88-96.

Trussel LO, Jackson MB (1985) Adenosine-activated potassium conductance in cultured striatal neurons. Proc Natl Acad Sci USA 82: 4857-4861.

Valverde MA, Diaz M, Sepulveda FV, Gill DR, Hyde SC, Higgins CF (1992) Volume-regulated chloride channels associated with the human multidrug-resistance P-glycoprotein. Nature 355:830-833.

Vasquez S, Rosenberg PA (1989) Extracellular 3':5'-cyclic nucleotide phosphodiesterase in rat cerebral cortex in dissociated cell culture. Soc Neurosci Abstr 15:168.

Weishaar RE, Burrows SD, Kobylarz DC, Quade MM, Evans DB (1986) Multiple molecular forms of cyclic nucleotide phosphodiesterase in cardiac and smooth muscle and in platelets. Biochem Pharmacol 35: 787-800.

White TD (1978) Release of ATP from a synaptosomal preparation by elevated extracellular $\mathrm{K}^{+}$and by veratridine. $\mathrm{J}$ Neurochem 30 : 329-336.

Williams M, Jarvis MF (1988) Adenosine antagonists as potential therapeutic agents. Pharmacol Biochem Behav 29:434-441.

Woodward DJ, Moises HC, Waterhouse BD, Hoffer BJ, Freedman R (1979) Modulatory actions of norepinephrine in the central nervous system. Fed Proc 38:2109-2116.

Wu C-F, Kelly LA, Butcher RW (1978) Cyclic AMP escape from fibroblasts. Adv Cyclic Nucleotide Res 9:781.

Wu PH, Phillis JW, Thierry DL (1982) Adenosine receptor agonists inhibit $\mathrm{K}^{+}$-evoked $\mathrm{Ca}^{2+}$ uptake by rat brain cortical synaptosomes. J Neurochem 39:700-708.

Yoon K-W, Rothman SM (1991) Adenosine inhibits excitatory but not inhibitory synaptic transmission in the hippocampus. J Neurosci 11:1375-1380. 